Supplement of Clim. Past, 14, 1881-1891, 2018

https://doi.org/10.5194/cp-14-1881-2018-supplement

C Author(s) 2018. This work is distributed under

the Creative Commons Attribution 4.0 License.

(c) (1)

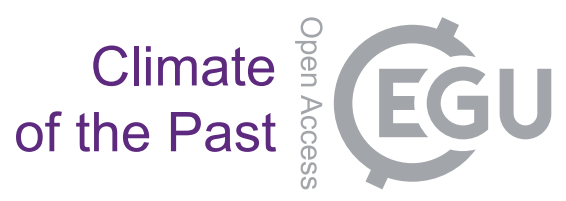

Supplement of

\title{
Hydro-climatic variability in the southwestern Indian Ocean between 6000 and 3000 years ago
}

\section{Hanying Li et al.}

Correspondence to: Hai Cheng (cheng021@xjtu.edu.cn)

The copyright of individual parts of the supplement might differ from the CC BY 4.0 License. 


\section{Supplementary Figures:}
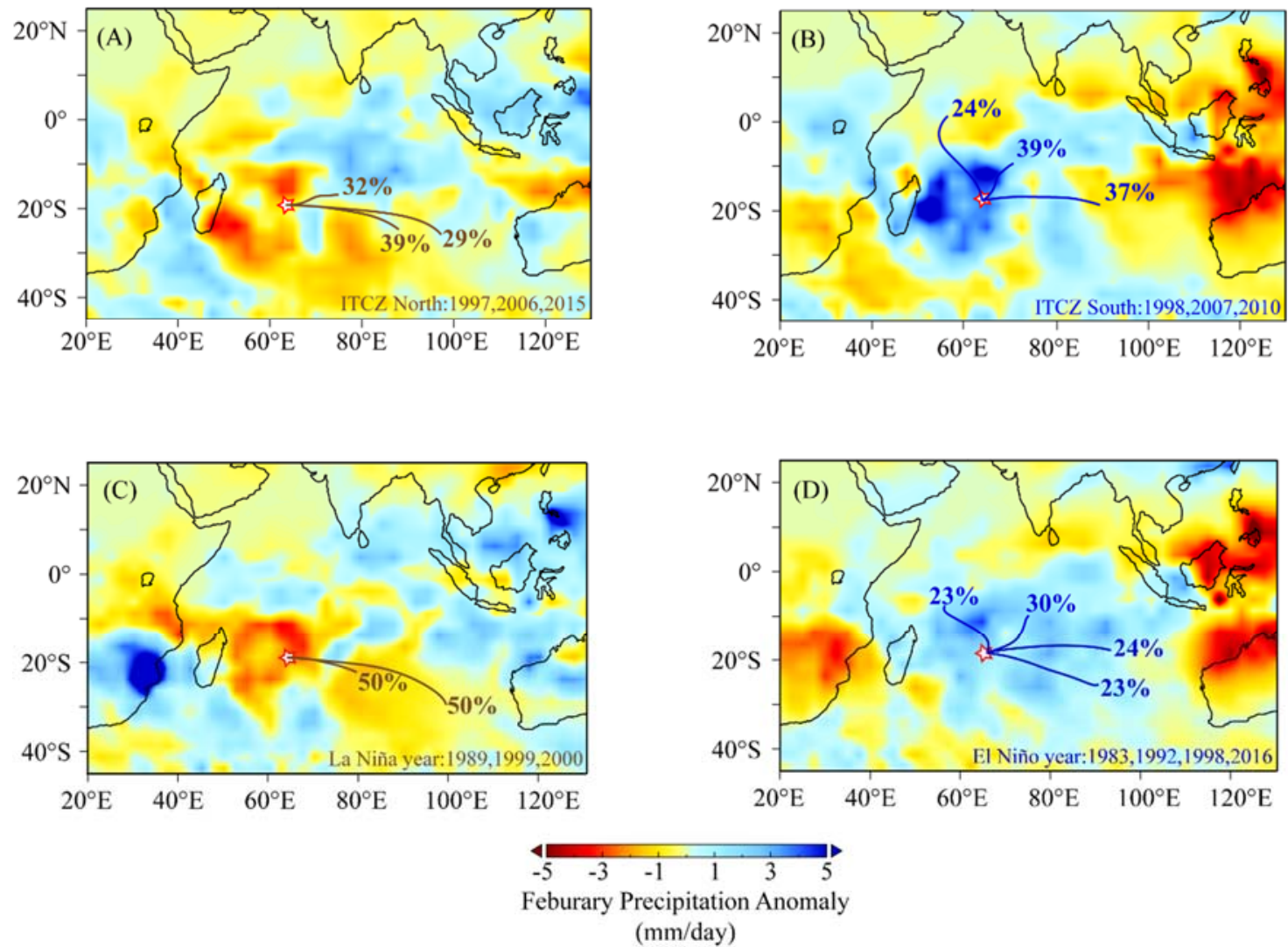

Supplementary Fig. 1. ITCZ and ENSO dynamics. (A and B) Spatial composite maps of precipitation anomalies for February (anomalies calculated with respect to the period 1981-2010) for the years marked by anomalous northward (A, 1997, 2006, 2015) and southward (B, 1998, 2007, 2010) locations of the southern boundary of the ITCZ (Lashkari et al., 2017; Freitas et al., 2017). The maps are overlain by backward (120 hours) low-level air parcel trajectory clusters and their relative contributions. (C and D) Same as in A and B but for La Niña (C, 1989, 1999, 2000) and El Niño (D, 1983, 1992, 1998, 2016) years. Precipitation data from GPCP (Adler et al., 2018). 


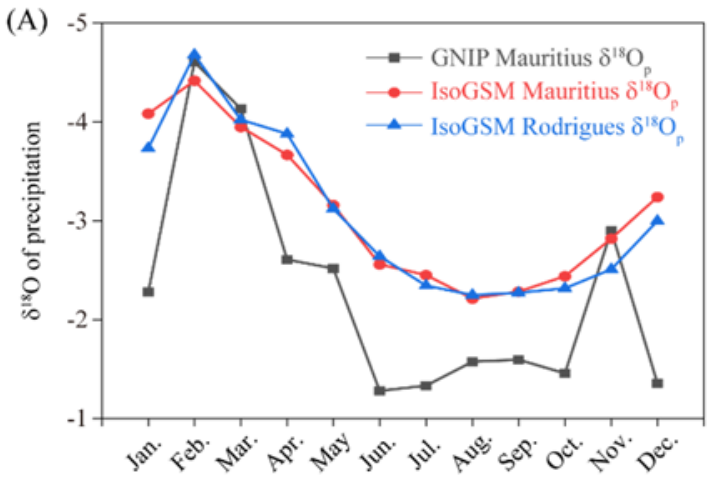

(B)

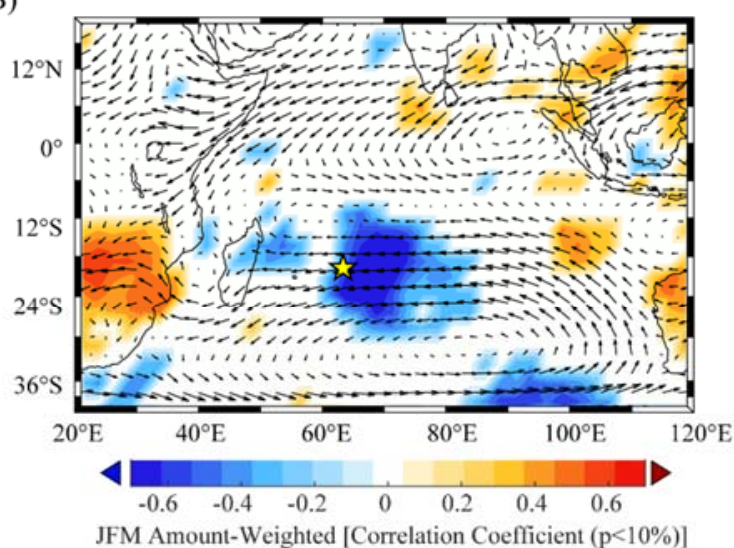

(C)

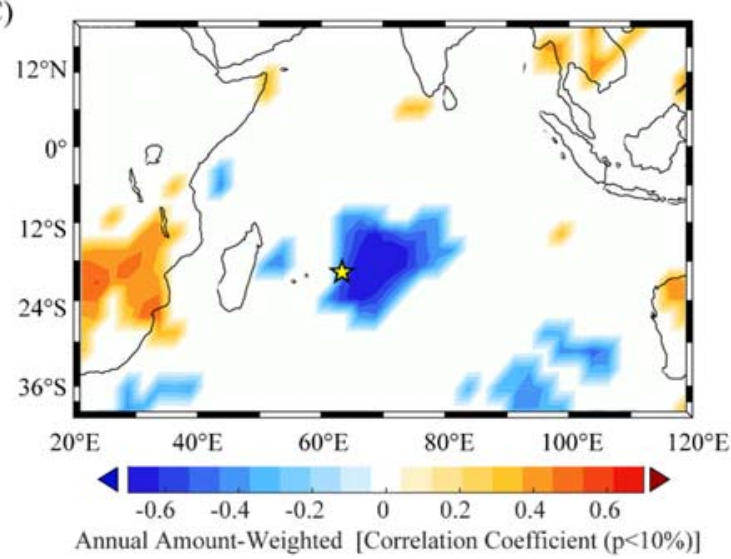

Supplementary Fig. 2. Modelled and observational data of $\delta^{18} \mathrm{O}$ in precipitation in the study area. (A) Monthly means of simulated $\delta^{18} \mathrm{O}_{\mathrm{p}}$ for Mauritius (red) and Rodrigues (blue) from IsoGSM (Yoshimura et al., 2008). Also shown are monthly means of $\delta^{18} \mathrm{O}_{\mathrm{p}}$ from six GNIP stations in Mauritius (black) covering the periods 19921995 and 2009-2014. (B and C) Spatial correlation maps for JFM (B) and annual (C) amount-weighted IsoGSM $\delta^{18} \mathrm{O}_{\mathrm{p}}$ from the nearest grid point to Rodrigues and the GPCP precipitation (GPCP v2.3) (Adler et al., 2018) for the period 1979 to 2016. 

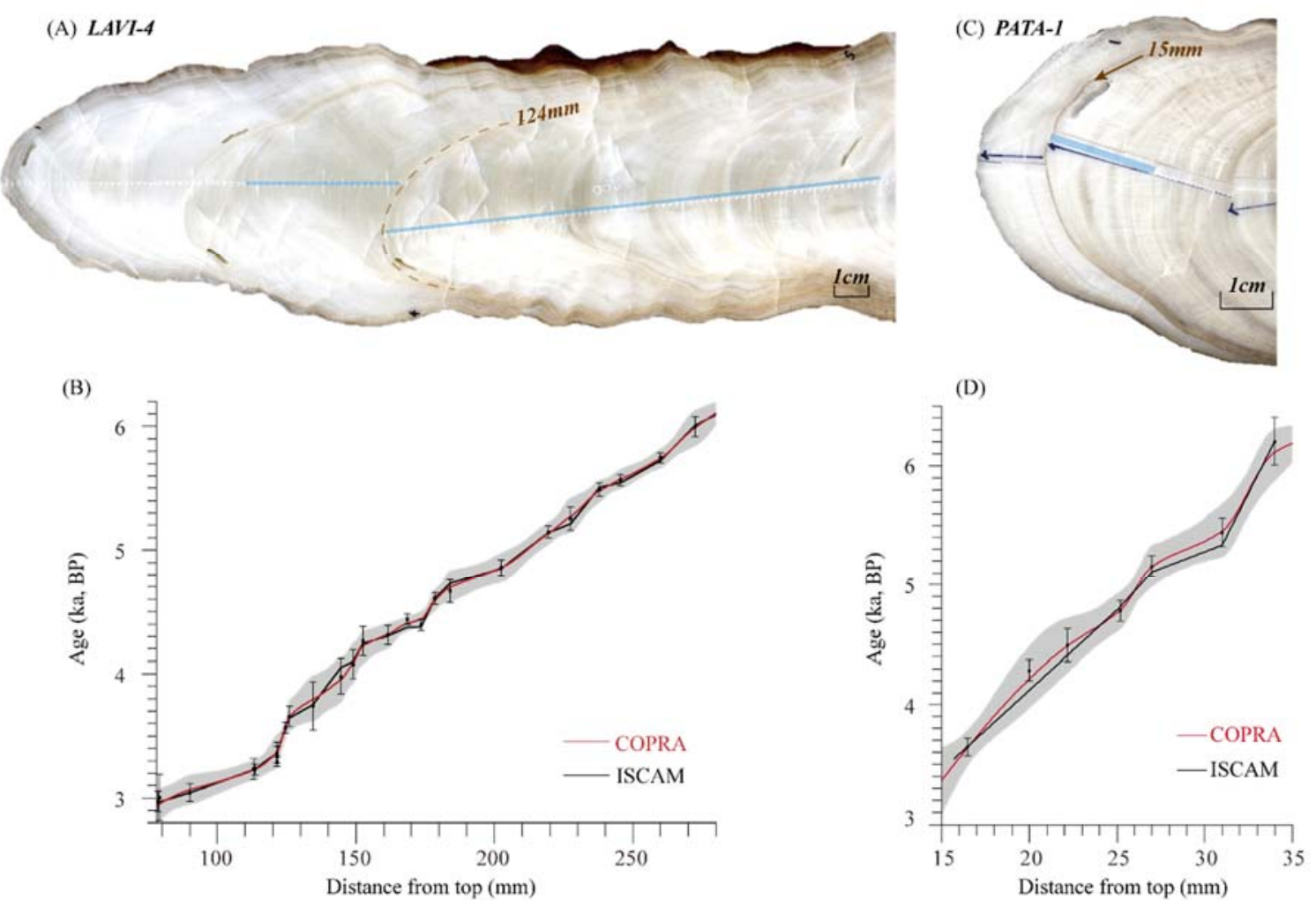

Supplementary Fig. 3. Age models of LAVI-4 and PATA-1 stalagmites. (A and C) scan pictures of stalagmite LAVI-4 and PATA-1, respectively. The blue bars line on the stalagmite slabs showing the stable isotope tracks. Dash line in A marks the layer at $124 \mathrm{~mm}$. Arrow in $\mathbf{C}$ marks the layer at $15 \mathrm{~mm}$. (B) LAVI-4 age models and age uncertainties obtained using COPRA (Breitenbach et al., 2012) (red) and ISCAM (Fohlmeister, 2012) (black). The gray band depicts the $95 \%$ confidence interval from COPRA. Error bars on ${ }^{230}$ Th dates represent $2 \sigma$ analytical errors. (D) Same as in (B) but for sample PATA-1. 
(A)

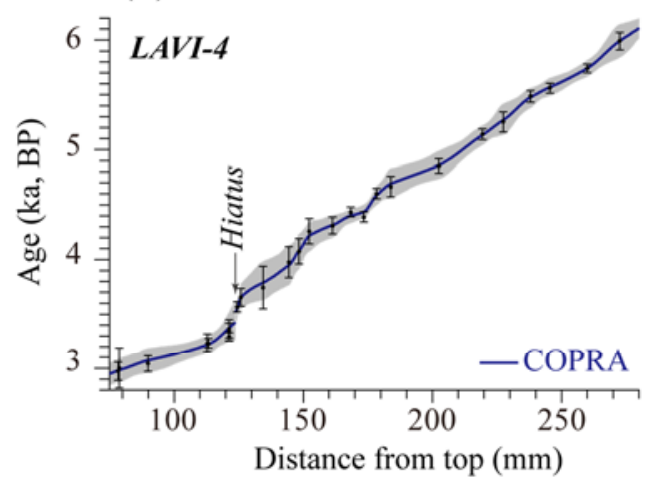

(C)

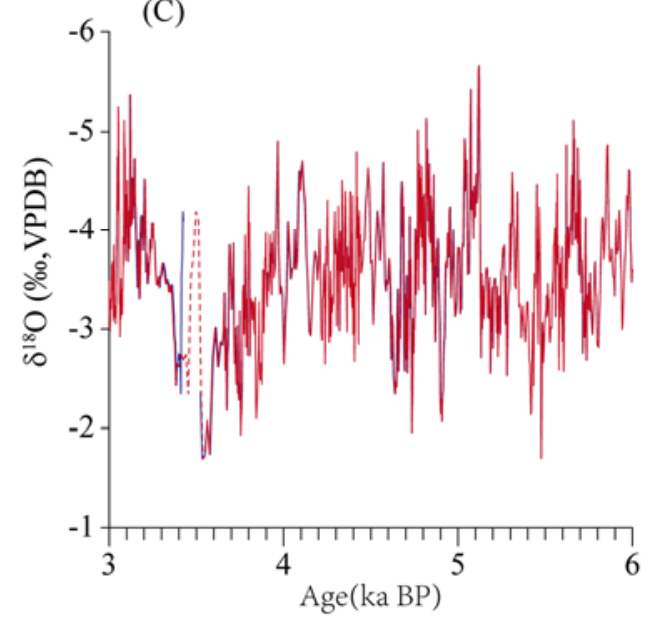

(B)

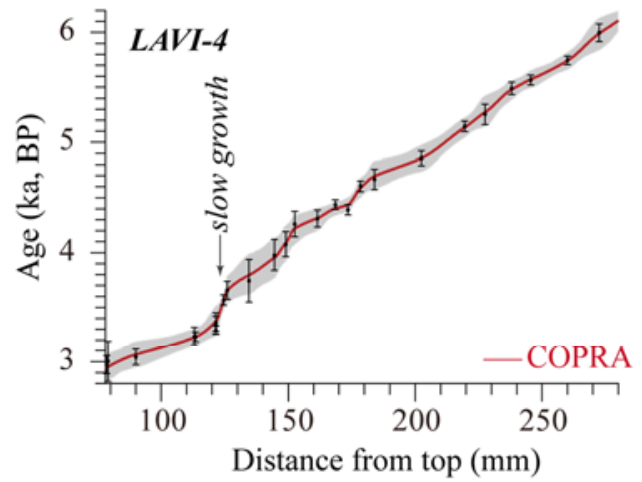

(D)

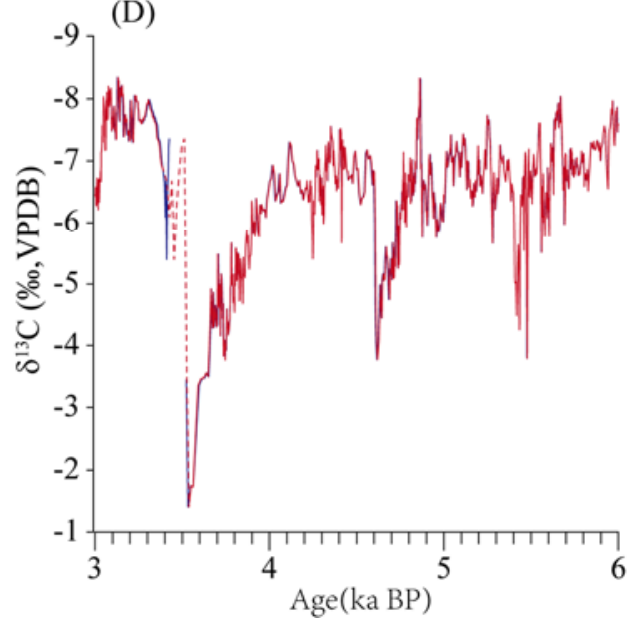

\section{Supplementary Fig. 4. Comparison of COPRA age model results. (A and B)}

COPRA age models (Breitenbach et al., 2012) of LAVI-4 with a hiatus at $124 \mathrm{~mm}$ (A) and no hiatus (B). (C) $\delta^{18} \mathrm{O}$ time series based on the age models in $\mathbf{A}$ and $\mathbf{B}$. (D) $\delta^{13} \mathrm{C}$ time series based on the age models in $\mathbf{A}$ and $\mathbf{B}$. The blue and red lines are the age model results from $\mathbf{A}$ and $\mathbf{B}$, respectively. There is a small offset between the two models, except for the period between 3.55 and 3.4 ka BP marked by red dashed lines. The main hydroclimate variations between 6 and $3 \mathrm{ka} \mathrm{BP}$ are robust irrespective of the age model used.

\section{References:}

Adler, R. F., Sapiano, M. R., Huffman, G. J., Wang, J. J., Gu, G., Bolvin, D., Chiu, L., Schneider, U., Becker, A., Nelkin, E., Xie, p., Ferraro, R., Shin, D.: The Global Precipitation Climatology Project (GPCP) monthly analysis (New Version 2.3) and a review of 2017 global precipitation, Atmosphere, 9, 138, 2018.

Breitenbach, S.F.M., Rehfeld, K., Goswami, B., Baldini, J.U.L., Ridley, H.E., Kennett, D.J., Prufer, K.M., Aquino, V.V., Asmerom, Y., Polyak, V.J., Cheng, H., Kurths, J., and Marwan, N.: Constructing Proxy Records from Age models (COPRA), Climate of the Past, 8, 1765-1779, 2012.

Fohlmeister, J.: A statistical approach to construct composite climate records of dated archives, Quaternary Geochronology, 14, 48-56, 2012.

Freitas, A. C. V., Aímola, L., Ambrizzi, T., and de Oliveira, C. P.: Extreme Intertropical Convergence Zone shifts over Southern Maritime Continent, Atmospheric Science Letters, 18, 2-10, 2017. 
Jaffey, A. H., Flynn, K. F., Glendenin, L. E., Bentley, W. T., and Essling, A. M.: Precision measurement of half-lives and specific activities of ${ }^{235} \mathrm{U}$ and ${ }^{238} \mathrm{U}$, Physical Review C, 4, 1889, https://doi.org/10.1103/PhysRevC.4.1889, 1971

Lashkari, H., Mohammadi, Z., and Keikhosravi, G.: Annual fluctuations and displacements of Inter Tropical Convergence Zone (ITCZ) within the range of Atlantic Ocean-India, Open Journal of Ecology, 7, 12, 2017.

Yoshimura, K., Kanamitsu, M., Noone, D., and Oki, T.: Historical isotope simulation using reanalysis atmospheric data, Journal of Geophysical Research: Atmospheres, 113, 2008. 


\section{Supplementary Tables}

Table S1

${ }^{230} \mathrm{Th}$ dating results. The error is $2 \mathrm{~s}$ error.

\begin{tabular}{|c|c|c|c|c|c|c|c|c|c|c|}
\hline $\begin{array}{l}\text { Sample } \\
\text { Number }\end{array}$ & $\operatorname{dft}(\mathbf{m m})$ & $\begin{array}{l}{ }^{238} \mathrm{U} \\
(\mathrm{ppb})\end{array}$ & $\begin{array}{l}{ }^{232} \mathrm{Th} \\
\text { (ppt) }\end{array}$ & $\begin{array}{l}{ }^{230} \mathrm{Th} /{ }^{232} \mathrm{Th} \\
\left(\text { atomic } \times 10^{-6} \text { ) }\right.\end{array}$ & $\begin{array}{c}\delta^{234} \mathrm{U}^{*} \\
\text { (measured) }\end{array}$ & $\begin{array}{c}{ }^{230} \mathrm{Th} /{ }^{238} \mathrm{U} \\
\text { (activity) }\end{array}$ & $\begin{array}{l}{ }^{230} \text { Th Age (yr) } \\
\text { (uncorrected) }\end{array}$ & $\begin{array}{c}{ }^{230} \text { Th Age (yr) } \\
\text { (corrected) }\end{array}$ & $\begin{array}{l}\delta^{234} U_{\text {Initial }} * * \\
\text { (corrected) }\end{array}$ & $\begin{array}{l}{ }^{230} \text { Th Age (yr } \\
\text { (corrected) }\end{array}$ \\
\hline \multicolumn{11}{|c|}{ LAVI-4 } \\
\hline LAVI4-78.5 & 78.5 & $91 \pm 0$ & $14 \pm 1$ & $3154 \pm 293$ & $65.9 \pm 1.3$ & $0.0293 \pm 0.0008$ & $3043 \pm 83$ & $3039 \pm 83$ & $66 \pm 1$ & $2972 \pm 83$ \\
\hline *LAVI4-80 & 79 & $72.4 \pm 0.1$ & $26 \pm 2$ & $1380 \pm 141$ & $69.8 \pm 2.3$ & $0.0298 \pm 0.0018$ & $3079 \pm 185$ & $3069 \pm 185$ & $70 \pm 2$ & $3002 \pm 185$ \\
\hline LAVI4-90 & 90 & $97.1 \pm 0.1$ & $33 \pm 1$ & $1479 \pm 66$ & $67.5 \pm 1.6$ & $0.0301 \pm 0.0007$ & $3118 \pm 72$ & $3109 \pm 73$ & $68 \pm 2$ & $3042 \pm 73$ \\
\hline LAVI4-113.5 & 113.5 & $136 \pm 0$ & $32 \pm 1$ & $2217 \pm 87$ & $65.4 \pm 1.3$ & $0.0318 \pm 0.0004$ & $3304 \pm 45$ & $3298 \pm 45$ & $66 \pm 1$ & $3231 \pm 45$ \\
\hline *LAVI4-113 & 113 & $148.8 \pm 0.2$ & $21 \pm 2$ & $3656 \pm 335$ & $69.0 \pm 1.8$ & $0.0319 \pm 0.0008$ & $3304 \pm 82$ & $3300 \pm 82$ & $70 \pm 2$ & $3233 \pm 82$ \\
\hline LAVI4-121.5 & 121.5 & $135.5 \pm 0.2$ & $19 \pm 2$ & $3881 \pm 369$ & $70.7 \pm 1.7$ & $0.0332 \pm 0.0008$ & $3434 \pm 84$ & $3430 \pm 84$ & $71 \pm 2$ & $3363 \pm 84$ \\
\hline *L4-123 & 121.5 & $117.2 \pm 0.2$ & $41 \pm 1$ & $1543 \pm 42$ & $67.0 \pm 1.5$ & $0.0325 \pm 0.0003$ & $3369 \pm 37$ & $3359 \pm 37$ & $68 \pm 2$ & $3292 \pm 37$ \\
\hline${ }^{*}$ L4-124-5 & 121.5 & $132.2 \pm 0.1$ & $24 \pm 2$ & $2979 \pm 221$ & $68.9 \pm 1.3$ & $0.0329 \pm 0.0008$ & $3406 \pm 81$ & $3401 \pm 81$ & $70 \pm 1$ & $3334 \pm 81$ \\
\hline LAVI-4-4 & 124.5 & $70.9 \pm 0.1$ & $15 \pm 2$ & $2747 \pm 317$ & $69.1 \pm 2.4$ & $0.0351 \pm 0.0004$ & $3639 \pm 46$ & $3634 \pm 46$ & $70 \pm 2$ & $3567 \pm 46$ \\
\hline L4-124-6 & 126 & $76.9 \pm 0.1$ & $95 \pm 2$ & $480 \pm 14$ & $66.7 \pm 1.7$ & $0.0361 \pm 0.0007$ & $3755 \pm 78$ & $3721 \pm 81$ & $67 \pm 2$ & $3654 \pm 81$ \\
\hline L4-134.5 & 134.5 & $49.1 \pm 0.0$ & $45 \pm 2$ & $687 \pm 44$ & $117.1 \pm 1.2$ & $0.0386 \pm 0.0019$ & $3834 \pm 194$ & $3810 \pm 195$ & $118 \pm 1$ & $3743 \pm 195$ \\
\hline L4-144.5 & 144.5 & $66.8 \pm 0.1$ & $170 \pm 4$ & $257 \pm 10$ & $72.3 \pm 1.5$ & $0.0397 \pm 0.0013$ & $4113 \pm 133$ & $4044 \pm 142$ & $73 \pm 2$ & $3977 \pm 142$ \\
\hline L4-1 & 148.5 & $66.7 \pm 0.1$ & $113 \pm 1$ & $393 \pm 11$ & $67.5 \pm 1.3$ & $0.0403 \pm 0.0010$ & $4190 \pm 110$ & $4144 \pm 115$ & $68 \pm 1$ & $4077 \pm 115$ \\
\hline L4-152.5 & 152.5 & $76.6 \pm 0.1$ & $39 \pm 2$ & $1362 \pm 65$ & $72.3 \pm 1.6$ & $0.0419 \pm 0.0011$ & $4340 \pm 115$ & $4326 \pm 116$ & $73 \pm 2$ & $4259 \pm 116$ \\
\hline L4-161.5 & 161.5 & $91.0 \pm 0.1$ & $74 \pm 2$ & $864 \pm 26$ & $71.3 \pm 1.6$ & $0.0424 \pm 0.0007$ & $4399 \pm 76$ & $4377 \pm 77$ & $72 \pm 2$ & $4310 \pm 77$ \\
\hline LAVI4-168.5 & 168.5 & $131 \pm 0$ & $29 \pm 1$ & $3273 \pm 126$ & $71.0 \pm 1.4$ & $0.0434 \pm 0.0004$ & $4507 \pm 43$ & $4501 \pm 43$ & $72 \pm 1$ & $4434 \pm 43$ \\
\hline L4-173.5 & 173.5 & $131.3 \pm 0.1$ & $31 \pm 1$ & $3030 \pm 111$ & $70.6 \pm 1.3$ & $0.0429 \pm 0.0004$ & $4462 \pm 46$ & $4456 \pm 46$ & $71 \pm 1$ & $4389 \pm 46$ \\
\hline L4-180-8 & 178.5 & $137.7 \pm 0.2$ & $21 \pm 1$ & $4778 \pm 234$ & $69.1 \pm 1.4$ & $0.0449 \pm 0.0004$ & $4673 \pm 48$ & $4669 \pm 48$ & $70 \pm 1$ & $4602 \pm 48$ \\
\hline L4-2 & 184 & $84.9 \pm 0.1$ & $94 \pm 1$ & $679 \pm 15$ & $70.2 \pm 1.3$ & $0.0458 \pm 0.0008$ & $4762 \pm 90$ & $4732 \pm 93$ & $71 \pm 1$ & $4665 \pm 93$ \\
\hline L4-3 & 202.5 & $113.6 \pm 0.1$ & $36 \pm 1$ & $2471 \pm 92$ & $70.5 \pm 1.2$ & $0.0473 \pm 0.0006$ & $4929 \pm 66$ & $4920 \pm 66$ & $71 \pm 1$ & $4853 \pm 66$ \\
\hline LAVI4-209 & 219.5 & $120 \pm 0$ & $47 \pm 1$ & $2098 \pm 62$ & $72.1 \pm 1.2$ & $0.0501 \pm 0.0004$ & $5219 \pm 47$ & $5209 \pm 48$ & $73 \pm 1$ & $5142 \pm 48$ \\
\hline L4-4 & 227.5 & $76.2 \pm 0.1$ & $44 \pm 1$ & $1475 \pm 51$ & $69.9 \pm 1.2$ & $0.0511 \pm 0.0009$ & $5336 \pm 92$ & $5320 \pm 93$ & $71 \pm 1$ & $5253 \pm 93$ \\
\hline LAVI4-228 & 238 & $108 \pm 0$ & $61 \pm 2$ & $1551 \pm 43$ & $72.5 \pm 1.2$ & $0.0534 \pm 0.0005$ & $5570 \pm 53$ & $5555 \pm 55$ & $74 \pm 1$ & $5488 \pm 55$ \\
\hline L4-251 & 245.5 & $93.9 \pm 0.1$ & $56 \pm 1$ & $1495 \pm 36$ & $75.0 \pm 1.4$ & $0.0543 \pm 0.0004$ & $5647 \pm 44$ & $5631 \pm 46$ & $76 \pm 1$ & $5564 \pm 46$ \\
\hline LAVI-4-5 & 260 & $131.7 \pm 0.2$ & $45 \pm 2$ & $2710 \pm 103$ & $74.6 \pm 2.1$ & $0.0559 \pm 0.0003$ & $5820 \pm 38$ & $5811 \pm 39$ & $76 \pm 2$ & $5744 \pm 39$ \\
\hline LAVI4-262.5 & 272.5 & $76 \pm 0$ & $109 \pm 2$ & $672 \pm 17$ & $73.0 \pm 1.3$ & $0.0584 \pm 0.0007$ & $6100 \pm 75$ & $6061 \pm 80$ & $74 \pm 1$ & $5994 \pm 80$ \\
\hline
\end{tabular}


${ }^{230}$ Th dating results. The error is $2 \mathrm{~s}$ error.

\begin{tabular}{|c|c|c|c|c|c|c|c|c|c|c|}
\hline $\begin{array}{l}\text { Sample } \\
\text { Number }\end{array}$ & $\operatorname{dft}(\mathbf{m m})$ & $\begin{array}{l}{ }^{238} \mathrm{U} \\
(\mathrm{ppb})\end{array}$ & $\begin{array}{l}{ }^{232} \mathrm{Th} \\
\text { (ppt) }\end{array}$ & $\begin{array}{r}{ }^{230} \mathrm{Th} /{ }^{232} \mathrm{Th} \\
\left(\text { atomic } \times 10^{-6}\right)\end{array}$ & $\begin{array}{c}\delta^{234} \mathrm{U}^{*} \\
\text { (measured) }\end{array}$ & $\begin{array}{l}{ }^{230} \mathrm{Th} /{ }^{238} \mathrm{U} \\
\text { (activity) }\end{array}$ & $\begin{array}{l}{ }^{230} \text { Th Age (yr) } \\
\text { (uncorrected) }\end{array}$ & $\begin{array}{c}{ }^{230} \text { Th Age (yr) } \\
\text { (corrected) }\end{array}$ & $\begin{array}{l}\delta^{234} U_{\text {Initial }}{ }^{* *} \\
\text { (corrected) }\end{array}$ & $\begin{array}{c}{ }^{230} \text { Th Age (yr BP)*** } \\
\text { (corrected) }\end{array}$ \\
\hline & & & & & PATA-1 & & & & & \\
\hline PATA-1-1 & 16.5 & $182.9 \pm 0.2$ & $506 \pm 10$ & $226 \pm 6$ & $108.8 \pm 1.7$ & $0.0379 \pm 0.0005$ & $3786 \pm 55$ & $3713 \pm 75$ & $110 \pm 2$ & $3650 \pm 75$ \\
\hline P1-20 & 20 & $198.7 \pm 0.3$ & $848 \pm 17$ & $171 \pm 4$ & $105.7 \pm 1.3$ & $0.0444 \pm 0.0004$ & $4464 \pm 36$ & $4352 \pm 87$ & $107 \pm 1$ & $4284 \pm 87$ \\
\hline P1-22.2 & 22.2 & $203.8 \pm 0.3$ & $1455 \pm 29$ & $109 \pm 2$ & $105.3 \pm 1.6$ & $0.0471 \pm 0.0004$ & $4750 \pm 38$ & $4562 \pm 138$ & $107 \pm 2$ & $4494 \pm 138$ \\
\hline A1-1 & 25.2 & $169 \pm 0.2$ & $636 \pm 13$ & $215 \pm 5$ & $107.5 \pm 1.4$ & $0.0492 \pm 0.0005$ & $4947 \pm 49$ & $4848 \pm 85$ & $109 \pm 1$ & $4784 \pm 85$ \\
\hline P1-27 & 27 & $143.4 \pm 0.2$ & $553 \pm 11$ & $226 \pm 5$ & $107.4 \pm 1.8$ & $0.0528 \pm 0.0005$ & $5327 \pm 56$ & $5225 \pm 91$ & $109 \pm 2$ & $5158 \pm 91$ \\
\hline P1-31 & 31 & $164.1 \pm 0.3$ & $961 \pm 19$ & $158 \pm 3$ & $107.4 \pm 2.1$ & $0.0561 \pm 0.0005$ & $5665 \pm 48$ & $5511 \pm 119$ & $109 \pm 2$ & $5444 \pm 119$ \\
\hline PATA1-34 & 34 & $134.4 \pm 0.2$ & $1421 \pm 29$ & $101 \pm 2$ & $104.8 \pm 1.4$ & $0.0645 \pm 0.0005$ & $6553 \pm 50$ & $6275 \pm 203$ & $107 \pm 1$ & $6209 \pm 203$ \\
\hline
\end{tabular}

U decay constants: $\lambda_{238}=1.55125 \times 10^{-10}$ (Jaffey et al., 1971) and $\lambda_{234}=2.82206 \times 10^{-6}$ (Cheng et al., 2013).

Th decay constant: $\lambda_{230}=9.1705 \times 10^{-6}$ (Cheng et al., 2013).

$* \delta^{234} U=\left(\left[{ }^{234} \mathrm{U} /{ }^{238} \mathrm{U}\right]_{\text {activity }}-1\right) \times 1000 . * * \delta^{234} \mathrm{U}_{\text {initial }}$ was calculated based on ${ }^{230}$ Th age $(\mathrm{T})$, i.e., $\delta^{234} \mathrm{U}_{\text {initial }}=\delta^{234} \mathrm{U}_{\text {measured }} \mathrm{x} \mathrm{e}^{\lambda 234 \times \mathrm{T}}$.

Corrected ${ }^{230} \mathrm{Th}$ ages assume the initial ${ }^{230} \mathrm{Th} /{ }^{232} \mathrm{Th}$ atomic ratio of $4.4 \pm 2.2 \times 10^{-6}$. Those are the values for a material at secular

equilibrium, with the bulk earth ${ }^{232} \mathrm{Th} /{ }^{238} \mathrm{U}$ value of 3.8. The errors are arbitrarily assumed to be $50 \%$.

***B.P. stands for "Before Present" where the "Present" is defined as the year 1950 A.D.

Sample numbers started with * are dated for replication, not used for age model. 


\begin{tabular}{|c|c|c|c|c|}
\hline DFT(mm) & $\begin{array}{c}\text { Copra_Age } \\
\text { (yr BP) }\end{array}$ & $\delta^{13} \mathrm{C}(\%)$ & $\delta^{18} \mathrm{O}(\%)$ & $\begin{array}{c}\text { Iscam_Age } \\
\text { (yr BP) }\end{array}$ \\
\hline 81 & 2999.467 & -6.446 & -3.485 & 2981 \\
\hline 81.2 & 3001.092 & -6.428 & -3.129 & 2982 \\
\hline 81.4 & 3002.737 & -6.576 & -3.103 & 2983 \\
\hline 81.6 & 3004.403 & -6.447 & -2.979 & 2985 \\
\hline 81.8 & 3006.087 & -6.289 & -3.082 & 2986 \\
\hline 82 & 3007.788 & -6.271 & -3.292 & 2988 \\
\hline 82.2 & 3009.504 & -6.331 & -3.305 & 2989 \\
\hline 82.4 & 3011.234 & -6.685 & -3.205 & 2990 \\
\hline 82.6 & 3012.977 & -6.722 & -3.446 & 2992 \\
\hline 82.8 & 3014.73 & -6.645 & -3.651 & 2993 \\
\hline 83 & 3016.493 & -6.651 & -3.489 & 2994 \\
\hline 83.2 & 3018.264 & -6.207 & -3.391 & 2996 \\
\hline 83.4 & 3020.041 & -6.51 & -3.678 & 2997 \\
\hline 83.6 & 3021.823 & -6.699 & -3.373 & 2998 \\
\hline 83.8 & 3023.609 & -6.329 & -3.076 & 3000 \\
\hline 84 & 3025.396 & -6.511 & -3.625 & 3001 \\
\hline 84.2 & 3027.184 & -6.709 & -3.85 & 3002 \\
\hline 84.4 & 3028.971 & -6.657 & -3.592 & 3004 \\
\hline 84.6 & 3030.755 & -6.724 & -3.116 & 3005 \\
\hline 84.8 & 3032.536 & -6.838 & -3.232 & 3007 \\
\hline 85 & 3034.311 & -6.663 & -3.233 & 3008 \\
\hline 85.2 & 3036.079 & -7.133 & -3.14 & 3009 \\
\hline 85.4 & 3037.838 & -7.147 & -3.054 & 3011 \\
\hline 85.6 & 3039.588 & -7.187 & -3.102 & 3012 \\
\hline 85.8 & 3041.325 & -7.279 & -3.183 & 3013 \\
\hline 86 & 3043.051 & -7.569 & -3.798 & 3015 \\
\hline 86.2 & 3044.761 & -7.305 & -4.442 & 3016 \\
\hline 86.4 & 3046.456 & -7.7 & -4.746 & 3017 \\
\hline 86.6 & 3048.133 & -8.032 & -4.205 & 3019 \\
\hline 86.8 & 3049.791 & -7.955 & -4.412 & 3020 \\
\hline 87 & 3051.43 & -7.906 & -5.251 & 3021 \\
\hline 87.2 & 3053.046 & -7.876 & -5.181 & 3023 \\
\hline 87.4 & 3054.638 & -7.796 & -4.663 & 3024 \\
\hline 87.6 & 3056.206 & -7.638 & -4.084 & 3026 \\
\hline 87.8 & 3057.748 & -7.169 & -3.246 & 3027 \\
\hline 88 & 3059.261 & -7.364 & -3.451 & 3028 \\
\hline 88.2 & 3060.746 & -7.389 & -3.331 & 3030 \\
\hline 88.4 & 3062.199 & -7.177 & -2.922 & 3031 \\
\hline 88.6 & 3063.62 & -7.712 & -3.15 & 3032 \\
\hline 88.8 & 3065.007 & -7.974 & -3.098 & 3034 \\
\hline 89 & 3066.359 & -7.904 & -3.117 & 3035 \\
\hline 89.2 & 3067.675 & -8.07 & -3.505 & 3036 \\
\hline 89.4 & 3068.952 & -8.107 & -3.439 & 3038 \\
\hline 89.6 & 3070.189 & -7.708 & -3.237 & 3039 \\
\hline 89.8 & 3071.385 & -7.661 & -3.189 & 3040 \\
\hline
\end{tabular}




$\begin{array}{ccccc}90 & 3072.538 & -7.814 & -3.268 & 3042 \\ 90.2 & 3073.669 & -7.672 & -3.22 & 3043 \\ 90.4 & 3074.798 & -7.909 & -3.146 & 3045 \\ 90.6 & 3075.927 & -8.036 & -3.257 & 3047 \\ 90.8 & 3077.055 & -8.203 & -3.408 & 3048 \\ 91 & 3078.182 & -8.158 & -3.389 & 3050 \\ 91.2 & 3079.309 & -7.922 & -3.926 & 3052 \\ 91.4 & 3080.435 & -8.031 & -3.866 & 3053 \\ 91.6 & 3081.561 & -7.902 & -3.644 & 3055 \\ 91.8 & 3082.686 & -7.883 & -3.984 & 3056 \\ 92 & 3083.811 & -8.129 & -4.021 & 3058 \\ 92.2 & 3084.937 & -7.93 & -4.266 & 3060 \\ 92.4 & 3086.062 & -8.025 & -5.113 & 3061 \\ 92.6 & 3087.188 & -8.123 & -4.881 & 3063 \\ 92.8 & 3088.314 & -8.138 & -3.913 & 3064 \\ 93 & 3089.44 & -8.004 & -3.863 & 3066 \\ 93.2 & 3090.567 & -8.151 & -4.464 & 3068 \\ 93.4 & 3091.695 & -8.167 & -4.835 & 3069 \\ 93.6 & 3092.823 & -8.018 & -4.16 & 3071 \\ 93.8 & 3093.952 & -7.817 & -3.917 & 3073 \\ 94 & 3095.083 & -7.666 & -3.752 & 3074 \\ 94.2 & 3096.214 & -7.546 & -3.69 & 3076 \\ 94.4 & 3097.347 & -7.482 & -3.809 & 3077 \\ 94.6 & 3098.481 & -7.607 & -4.098 & 3079 \\ 94.8 & 3099.616 & -7.572 & -4.223 & 3081 \\ 95 & 3100.753 & -7.587 & -4.366 & 3082 \\ 95.2 & 3101.892 & -7.704 & -4.205 & 3084 \\ 95.4 & 3103.033 & -7.569 & -4.323 & 3085 \\ 95.6 & 3104.175 & -7.527 & -4.508 & 3087 \\ 95.8 & 3105.32 & -7.675 & -4.509 & 3089 \\ 96 & 3106.466 & -7.788 & -4.376 & 3090 \\ 96.2 & 3107.615 & -7.8 & -4.088 & 3092 \\ 96.4 & 3108.767 & -7.865 & -3.892 & 3093 \\ 96.6 & 3109.92 & -7.798 & -3.683 & 3095 \\ 96.8 & 3111.077 & -7.879 & -3.737 & 3097 \\ 97 & 3112.236 & -7.797 & -3.838 & 3098 \\ 97.2 & 3113.398 & -7.696 & -4.077 & 3100 \\ 97.4 & 3114.563 & -7.49 & -4.199 & 3102 \\ 97.6 & 3115.731 & -7.474 & -4.087 & 3103 \\ 97.8 & 3116.902 & -7.55 & -3.815 & 3105 \\ 98 & 3118.076 & -7.544 & -3.812 & 3106 \\ 98.2 & 3119.254 & -7.44 & -4.254 & 3108 \\ 98.4 & 3120.436 & -7.345 & -4.991 & 3110 \\ 98.6 & 3121.621 & -7.354 & -5.369 & 3111 \\ 98.8 & 3122.809 & -7.386 & -4.972 & 3113 \\ 99.2 & 3124.002 & -7.625 & -4.902 & 3114 \\ 99.6 & 3125.199 & -7.719 & -4.514 & 3116 \\ 90 & 3126.399 & -7.769 & -4.068 & 3118 \\ & 3128.814 & -7.965 & -4.049 & 3119 \\ 9130.027 & -8.08 & -4.121 & 3123\end{array}$




$\begin{array}{ccccc}100.2 & 3131.246 & -8.346 & -4.078 & 3124 \\ 100.4 & 3132.469 & -8.339 & -4.181 & 3126 \\ 100.6 & 3133.696 & -8.314 & -4.323 & 3127 \\ 100.8 & 3134.929 & -8.279 & -4.531 & 3129 \\ 101 & 3136.166 & -8.287 & -4.385 & 3131 \\ 101.2 & 3137.409 & -8.181 & -4.131 & 3132 \\ 101.4 & 3138.657 & -8.156 & -4.122 & 3134 \\ 101.6 & 3139.911 & -7.964 & -4.247 & 3135 \\ 101.8 & 3141.169 & -7.708 & -4.329 & 3137 \\ 102 & 3142.434 & -7.638 & -4.37 & 3139 \\ 102.2 & 3143.704 & -7.694 & -4.378 & 3140 \\ 102.4 & 3144.98 & -7.949 & -4.433 & 3142 \\ 102.6 & 3146.262 & -8.083 & -4.714 & 3143 \\ 102.8 & 3147.55 & -8.171 & -4.73 & 3145 \\ 103 & 3148.843 & -8.18 & -4.594 & 3147 \\ 103.2 & 3150.144 & -8.106 & -4.382 & 3148 \\ 103.4 & 3151.45 & -8.073 & -4.25 & 3150 \\ 103.6 & 3152.764 & -8.071 & -4.233 & 3152 \\ 103.8 & 3154.083 & -8.047 & -4.155 & 3153 \\ 104 & 3155.41 & -8.161 & -4.034 & 3155 \\ 104.2 & 3156.743 & -8.213 & -3.958 & 3156 \\ 104.4 & 3158.084 & -8.094 & -3.816 & 3158 \\ 104.6 & 3159.431 & -8.051 & -3.764 & 3160 \\ 104.8 & 3160.786 & -8.069 & -3.857 & 3161 \\ 105 & 3162.147 & -7.97 & -3.653 & 3163 \\ 105.2 & 3163.517 & -7.875 & -3.543 & 3164 \\ 105.4 & 3164.894 & -7.594 & -3.42 & 3166 \\ 105.6 & 3166.278 & -7.39 & -3.479 & 3168 \\ 105.8 & 3167.67 & -7.401 & -3.566 & 3169 \\ 106 & 3169.07 & -7.452 & -3.707 & 3171 \\ 106.2 & 3170.478 & -7.629 & -3.893 & 3173 \\ 106.4 & 3171.894 & -7.677 & -3.742 & 3174 \\ 106.6 & 3173.319 & -7.721 & -3.624 & 3176 \\ 106.8 & 3174.752 & -7.73 & -3.486 & 3177 \\ 107 & 3176.193 & -7.742 & -3.304 & 3179 \\ 107.2 & 3177.642 & -7.669 & -3.329 & 3181 \\ 107.4 & 3179.101 & -7.628 & -3.508 & 3182 \\ 107.6 & 3180.568 & -7.641 & -3.687 & 3184 \\ 107.8 & 3182.044 & -7.672 & -3.976 & 3185 \\ 108 & 3183.529 & -7.542 & -3.924 & 3187 \\ 108.2 & 3185.023 & -7.513 & -4.11 & 3189 \\ 108.4 & 3186.527 & -7.46 & -4.14 & 3190 \\ 108.6 & 3188.04 & -7.42 & -4.155 & 3192 \\ 108.8 & 3189.562 & -7.462 & -4.143 & 3193 \\ 109 & 3191.094 & -7.477 & -4.021 & 3195 \\ 109.2 & 3192.635 & -7.444 & -4.106 & 3197 \\ 109.4 & 3194.187 & -7.48 & -3.939 & 3198 \\ 109.6 & 3195.748 & -7.355 & -3.902 & 3200 \\ 109.8 & 3197.319 & -7.317 & -3.797 & 3202 \\ 110 & 3198.901 & -7.384 & -3.916 & 3203 \\ 110.2 & 3200.493 & -7.328 & -3.923 & 3205\end{array}$




$\begin{array}{ccccc}110.4 & 3202.095 & -7.483 & -4.152 & 3206 \\ 110.6 & 3203.708 & -7.687 & -4.245 & 3208 \\ 110.8 & 3205.331 & -7.889 & -4.52 & 3210 \\ 111 & 3206.965 & -7.975 & -4.469 & 3211 \\ 111.2 & 3208.61 & -7.834 & -4.257 & 3213 \\ 111.4 & 3210.266 & -7.807 & -4.055 & 3214 \\ 111.6 & 3211.932 & -7.704 & -3.919 & 3216 \\ 111.8 & 3213.611 & -7.351 & -3.575 & 3218 \\ 112 & 3215.3 & -7.402 & -3.762 & 3219 \\ 112.2 & 3217.001 & -7.369 & -3.753 & 3221 \\ 112.4 & 3218.713 & -7.298 & -3.699 & 3223 \\ 112.6 & 3220.437 & -7.495 & -3.653 & 3224 \\ 112.8 & 3222.173 & -7.608 & -3.458 & 3226 \\ 113 & 3223.921 & -7.75 & -3.5 & 3227 \\ 113.2 & 3225.68 & -7.845 & -3.502 & 3229 \\ 113.4 & 3227.452 & -7.948 & -3.597 & 3231 \\ 113.6 & 3229.249 & -8.051 & -3.544 & 3233 \\ 113.8 & 3231.145 & -7.995 & -3.659 & 3236 \\ 114 & 3233.153 & -8.011 & -3.673 & 3240 \\ 114.2 & 3235.271 & -8.045 & -3.903 & 3243 \\ 114.4 & 3237.497 & -7.984 & -3.778 & 3246 \\ 114.6 & 3239.829 & -7.962 & -3.858 & 3250 \\ 114.8 & 3242.265 & -7.965 & -3.817 & 3253 \\ 115 & 3244.805 & -7.939 & -4.078 & 3256 \\ 115.2 & 3247.446 & -7.857 & -3.998 & 3260 \\ 115.4 & 3250.187 & -7.816 & -3.926 & 3263 \\ 115.6 & 3253.026 & -7.645 & -4.08 & 3266 \\ 115.8 & 3255.961 & -7.651 & -3.92 & 3270 \\ 116 & 3258.991 & -7.646 & -3.88 & 3273 \\ 116.2 & 3262.114 & -7.666 & -3.776 & 3276 \\ 116.4 & 3265.329 & -7.601 & -3.602 & 3280 \\ 116.6 & 3268.633 & -7.621 & -3.526 & 3283 \\ 116.8 & 3272.026 & -7.61 & -3.443 & 3286 \\ 117 & 3275.506 & -7.676 & -3.487 & 3290 \\ 117.2 & 3279.07 & -7.651 & -3.449 & 3293 \\ 117.4 & 3282.717 & -7.72 & -3.526 & 3296 \\ 117.6 & 3286.447 & -7.709 & -3.419 & 3300 \\ 117.8 & 3290.256 & -7.788 & -3.511 & 3303 \\ 118 & 3294.143 & -7.834 & -3.529 & 3306 \\ 118.2 & 3298.107 & -7.92 & -3.569 & 3309 \\ 118.4 & 3302.147 & -7.911 & -3.639 & 3313 \\ 118.6 & 3306.259 & -7.989 & -3.665 & 3316 \\ 118.8 & 3310.443 & -7.943 & -3.625 & 3319 \\ 119 & 3314.698 & -7.882 & -3.585 & 3323 \\ 119.2 & 3319.021 & -7.836 & -3.512 & 3326 \\ 119.4 & 3323.41 & -7.8 & -3.457 & 3329 \\ 119.6 & 3327.865 & -7.765 & -3.496 & 3333 \\ 119.8 & 3332.383 & -7.698 & -3.459 & 3336 \\ 120 & 3336.964 & -7.706 & -3.449 & 3339 \\ 120.2 & 3341.604 & -7.635 & -3.374 & 3343 \\ 120.4 & 3346.303 & -7.623 & -3.427 & 3346\end{array}$




$\begin{array}{ccccc}120.6 & 3351.059 & -7.568 & -3.372 & 3349 \\ 120.8 & 3355.87 & -7.377 & -3.444 & 3353 \\ 121 & 3360.735 & -7.337 & -3.295 & 3356 \\ 121.2 & 3365.652 & -7.314 & -3.138 & 3359 \\ 121.4 & 3370.619 & -7.229 & -2.927 & 3363 \\ 121.6 & 3375.891 & -7.059 & -2.859 & 3371 \\ 121.8 & 3382.915 & -6.928 & -2.43 & 3384 \\ 122 & 3391.722 & -6.848 & -2.648 & 3398 \\ 122.2 & 3402.075 & -6.76 & -2.618 & 3411 \\ 122.4 & 3413.741 & -6.644 & -2.75 & 3424 \\ 122.6 & 3426.482 & -6.092 & -2.688 & 3438 \\ 122.8 & 3440.065 & -6.672 & -2.741 & 3451 \\ 123 & 3454.252 & -5.398 & -2.345 & 3464 \\ 123.2 & 3468.809 & -6.268 & -3.52 & 3478 \\ 123.4 & 3483.499 & -6.806 & -3.734 & 3491 \\ 123.6 & 3498.089 & -7.244 & -4.195 & 3505 \\ 123.8 & 3512.341 & -7.354 & -4.1 & 3518 \\ 124 & 3526.021 & -3.446 & -2.352 & 3531 \\ 124.2 & 3538.893 & -1.408 & -1.695 & 3545 \\ 124.4 & 3550.722 & -1.744 & -1.726 & 3558 \\ 124.6 & 3561.898 & -1.727 & -2.081 & 3570 \\ 124.8 & 3575.584 & -2.49 & -1.739 & 3581 \\ 125 & 3591.226 & -3.353 & -2.707 & 3592 \\ 125.2 & 3607.601 & -3.468 & -3.014 & 3602 \\ 125.4 & 3623.49 & -3.472 & -2.62 & 3613 \\ 125.6 & 3637.67 & -3.56 & -2.868 & 3624 \\ 125.8 & 3648.92 & -3.499 & -2.834 & 3635 \\ 126 & 3656.018 & -3.93 & -2.999 & 3645 \\ 126.2 & 3660.585 & -4.379 & -3.365 & 3648 \\ 126.4 & 3665.055 & -4.921 & -2.582 & 3650 \\ 126.6 & 3669.429 & -4.426 & -3.005 & 3653 \\ 126.8 & 3673.711 & -4.284 & -2.181 & 3655 \\ 127 & 3677.902 & -4.858 & -2.722 & 3658 \\ 127.2 & 3682.004 & -4.301 & -2.86 & 3660 \\ 127.4 & 3686.019 & -4.442 & -3.697 & 3663 \\ 127.6 & 3689.948 & -4.653 & -3.855 & 3665 \\ 127.8 & 3693.796 & -4.444 & -3.733 & 3668 \\ 128 & 3697.562 & -4.302 & -3.461 & 3671 \\ 128.2 & 3701.249 & -4.343 & -3.2 & 3673 \\ 128.4 & 3704.859 & -5.478 & -3.34 & 3676 \\ 128.6 & 3708.395 & -4.625 & -3.577 & 3678 \\ 128.8 & 3711.857 & -4.824 & -3.872 & 3681 \\ 129 & 3715.249 & -4.99 & -2.941 & 3683 \\ 129.2 & 3718.572 & -4.16 & -2.652 & 3686 \\ 129.4 & 3721.828 & -5.16 & -3.012 & 3688 \\ 129.6 & 3725.019 & -5.099 & -2.668 & 3691 \\ 129.8 & 3728.147 & -4.868 & -2.439 & 3693 \\ 130 & 3731.215 & -4.441 & -2.547 & 3696 \\ 130.2 & 3734.223 & -4.676 & -3.032 & 3698 \\ 130.4 & 3737.175 & -3.833 & -2.354 & 3701 \\ 130.6 & 3740.072 & -4.032 & -2.394 & 3703\end{array}$




$\begin{array}{ccccc}130.8 & 3742.916 & -4.087 & -2.282 & 3706 \\ 131 & 3745.71 & -3.77 & -2.451 & 3708 \\ 131.2 & 3748.454 & -4.015 & -3.178 & 3711 \\ 131.4 & 3751.152 & -3.926 & -2.375 & 3713 \\ 131.6 & 3753.805 & -4.284 & -1.931 & 3716 \\ 131.8 & 3756.416 & -4.589 & -2.129 & 3718 \\ 132 & 3758.985 & -4.317 & -2.15 & 3721 \\ 132.2 & 3761.516 & -4.377 & -2.371 & 3723 \\ 132.4 & 3764.01 & -4.172 & -2.783 & 3726 \\ 132.6 & 3766.469 & -4.234 & -3.37 & 3728 \\ 132.8 & 3768.896 & -4.266 & -3.442 & 3731 \\ 133 & 3771.291 & -4.409 & -2.995 & 3734 \\ 133.2 & 3773.658 & -4.433 & -2.948 & 3736 \\ 133.4 & 3775.999 & -4.938 & -3.284 & 3739 \\ 133.6 & 3778.315 & -4.994 & -3.949 & 3741 \\ 133.8 & 3780.607 & -5.546 & -3.851 & 3744 \\ 134 & 3782.88 & -5.667 & -3.36 & 3746 \\ 134.2 & 3785.133 & -5.01 & -2.817 & 3749 \\ 134.4 & 3787.37 & -4.974 & -3.305 & 3751 \\ 134.6 & 3789.604 & -5.155 & -3.073 & 3755 \\ 134.8 & 3791.899 & -4.795 & -2.93 & 3761 \\ 135 & 3794.267 & -4.699 & -2.735 & 3767 \\ 135.2 & 3796.704 & -4.771 & -4.109 & 3773 \\ 135.4 & 3799.209 & -4.541 & -4.449 & 3779 \\ 135.6 & 3801.78 & -5.159 & -3.822 & 3785 \\ 135.8 & 3804.414 & -4.972 & -4.081 & 3791 \\ 136 & 3807.109 & -5.578 & -2.73 & 3797 \\ 136.2 & 3809.865 & -5.565 & -2.995 & 3803 \\ 136.4 & 3812.677 & -5.046 & -3.305 & 3809 \\ 136.6 & 3815.545 & -5.072 & -3.277 & 3815 \\ 136.8 & 3818.467 & -5.502 & -3.267 & 3821 \\ 137 & 3821.44 & -5.175 & -3.093 & 3827 \\ 137.2 & 3824.462 & -5.472 & -3.1 & 3833 \\ 137.4 & 3827.531 & -4.725 & -3.275 & 3839 \\ 137.6 & 3830.646 & -4.743 & -3.016 & 3845 \\ 137.8 & 3833.804 & -5.408 & -2.948 & 3851 \\ 138 & 3837.003 & -5.322 & -3.318 & 3857 \\ 138.2 & 3840.242 & -5.098 & -2.302 & 3863 \\ 138.4 & 3843.517 & -5.093 & -2.1 & 3868 \\ 138.6 & 3846.828 & -4.681 & -2.208 & 3874 \\ 138.8 & 3850.172 & -5.427 & -2.4 & 3880 \\ 139 & 3853.546 & -5.124 & -2.65 & 3886 \\ 139.2 & 3856.95 & -5.133 & -2.81 & 3892 \\ 139.4 & 3860.381 & -5.1 & -3.273 & 3898 \\ 139.6 & 3863.836 & -5.559 & -2.508 & 3904 \\ 139.8 & 3867.315 & -5.733 & -2.484 & 3910 \\ 140 & 3870.814 & -5.865 & -2.655 & 3916 \\ 140.2 & 3874.332 & -5.663 & -2.44 & 3922 \\ 140.4 & 3877.867 & -5.784 & -2.803 & 3928 \\ 140.6 & 3881.417 & -5.453 & -3.598 & 3934 \\ 140.8 & 3884.979 & -5.239 & -3.591 & 3940\end{array}$




$\begin{array}{ccccc}141 & 3888.552 & -5.444 & -2.961 & 3946 \\ 141.2 & 3892.134 & -5.469 & -3.718 & 3952 \\ 141.4 & 3895.722 & -5.645 & -3.71 & 3958 \\ 141.6 & 3899.315 & -6.349 & -3.976 & 3964 \\ 141.8 & 3902.911 & -6.253 & -3.339 & 3970 \\ 142 & 3906.507 & -5.815 & -3.23 & 3976 \\ 142.2 & 3910.101 & -5.894 & -3.573 & 3982 \\ 142.4 & 3913.692 & -6.012 & -3.384 & 3988 \\ 142.6 & 3917.277 & -5.773 & -3.516 & 3993 \\ 142.8 & 3920.855 & -5.863 & -3.956 & 3999 \\ 143 & 3924.423 & -6.388 & -3.733 & 4005 \\ 143.4 & 3931.522 & -6.126 & -3.348 & 4017 \\ 143.6 & 3935.049 & -5.844 & -3.869 & 4023 \\ 143.8 & 3938.558 & -5.794 & -3.67 & 4029 \\ 144 & 3942.047 & -5.766 & -3.986 & 4035 \\ 144.2 & 3945.514 & -6.286 & -3.517 & 4041 \\ 144.6 & 3952.487 & -6.146 & -3.631 & 4051 \\ 144.8 & 3956.74 & -6.29 & -3.821 & 4053 \\ 145 & 3961.753 & -6.225 & -4.245 & 4055 \\ 145.2 & 3967.445 & -6.188 & -4.906 & 4058 \\ 145.4 & 3973.736 & -6.216 & -3.793 & 4060 \\ 145.6 & 3980.546 & -6.082 & -3.35 & 4062 \\ 145.8 & 3987.797 & -6.379 & -3.429 & 4064 \\ 146 & 3995.406 & -6.515 & -3.343 & 4066 \\ 146.2 & 4003.296 & -6.673 & -2.649 & 4068 \\ 146.4 & 4011.385 & -6.71 & -3.072 & 4071 \\ 146.6 & 4019.594 & -6.925 & -3.382 & 4073 \\ 146.8 & 4027.843 & -6.794 & -4.089 & 4075 \\ 147 & 4036.051 & -6.35 & -3.788 & 4077 \\ 147.2 & 4044.14 & -6.434 & -3.507 & 4079 \\ 147.4 & 4052.029 & -6.502 & -3.606 & 4081 \\ 147.6 & 4059.638 & -6.792 & -3.621 & 4084 \\ 147.8 & 4066.887 & -6.32 & -4.003 & 4086 \\ 148 & 4073.697 & -6.355 & -3.606 & 4088 \\ 148.2 & 4079.986 & -6.342 & -3.687 & 4090 \\ 148.6 & 4090.87 & -6.506 & -4.603 & 4094 \\ 148.8 & 4096.575 & -6.576 & -4.41 & 4097 \\ 149 & 4102.889 & -6.601 & -4.605 & 4099 \\ 149.2 & 4109.726 & -7.027 & -4.706 & 4107 \\ 149.4 & 4117 & -7.297 & -4.432 & 4115 \\ 149.6 & 4124.624 & -7.242 & -4.261 & 4123 \\ 149.8 & 4132.513 & -7.005 & -3.76 & 4131 \\ 150 & 4140.58 & -6.947 & -3.473 & 4139 \\ 150.2 & 4148.739 & -6.749 & -2.978 & 4148 \\ 150.4 & 4156.904 & -6.673 & -2.933 & 4156 \\ 150.6 & 4164.99 & -6.59 & -3.275 & 4164 \\ 150.8 & 4172.909 & -6.686 & -3.602 & 4172 \\ 151 & 4180.576 & -6.473 & -3.784 & 4180 \\ 151.2 & 4187.905 & -6.527 & -3.724 & 4188 \\ 151.4 & 4194.81 & -6.373 & -3.497 & 4196 \\ 151.6 & 4201.204 & -6.474 & -3.649 & 4205\end{array}$




$\begin{array}{ccccc}151.8 & 4207.002 & -6.558 & -4.014 & 4213 \\ 152 & 4212.117 & -6.543 & -3.618 & 4221 \\ 152.2 & 4216.463 & -6.677 & -2.95 & 4229 \\ 152.4 & 4219.954 & -6.492 & -2.886 & 4237 \\ 152.6 & 4222.627 & -6.39 & -2.895 & 4242 \\ 152.8 & 4225.16 & -6.331 & -3.303 & 4243 \\ 153 & 4227.672 & -6.44 & -3.342 & 4245 \\ 153.2 & 4230.163 & -6.337 & -3.559 & 4246 \\ 153.4 & 4232.633 & -6.588 & -3.659 & 4247 \\ 153.6 & 4235.083 & -6.677 & -3.457 & 4249 \\ 153.8 & 4237.512 & -6.459 & -2.646 & 4250 \\ 154 & 4239.92 & -6.588 & -3.149 & 4251 \\ 154.2 & 4242.308 & -6.075 & -3.24 & 4253 \\ 154.4 & 4244.675 & -5.956 & -3.835 & 4254 \\ 154.6 & 4247.022 & -5.769 & -3.645 & 4255 \\ 154.8 & 4249.349 & -5.407 & -4.027 & 4257 \\ 155 & 4251.655 & -5.937 & -4.308 & 4258 \\ 155.2 & 4253.941 & -6.352 & -3.921 & 4259 \\ 155.4 & 4256.207 & -6.258 & -3.842 & 4261 \\ 155.6 & 4258.453 & -6.137 & -3.56 & 4262 \\ 155.8 & 4260.679 & -6.381 & -2.866 & 4263 \\ 156 & 4262.885 & -6.54 & -2.871 & 4265 \\ 156.2 & 4265.071 & -6.922 & -3.203 & 4266 \\ 156.4 & 4267.238 & -6.739 & -3.336 & 4268 \\ 156.6 & 4269.385 & -6.905 & -3.578 & 4269 \\ 156.8 & 4271.512 & -6.86 & -2.926 & 4270 \\ 157 & 4273.62 & -7.166 & -3.257 & 4272 \\ 157.2 & 4275.708 & -7.041 & -3.311 & 4273 \\ 157.4 & 4277.777 & -6.862 & -3.282 & 4274 \\ 157.6 & 4279.826 & -6.935 & -3.171 & 4276 \\ 157.8 & 4281.856 & -6.938 & -3.34 & 4277 \\ 158 & 4283.868 & -7.029 & -3.254 & 4278 \\ 158.2 & 4285.859 & -6.353 & -3.263 & 4280 \\ 158.4 & 4287.832 & -6.706 & -3.57 & 4281 \\ 158.6 & 4289.786 & -6.765 & -3.928 & 4282 \\ 158.8 & 4291.721 & -6.435 & -3.992 & 4284 \\ 159 & 4293.637 & -6.589 & -4.193 & 4285 \\ 159.2 & 4295.535 & -6.294 & -3.93 & 4286 \\ 159.4 & 4297.414 & -6.629 & -3.764 & 4288 \\ 159.6 & 4299.274 & -6.721 & -3.91 & 4289 \\ 159.8 & 4301.115 & -6.279 & -3.815 & 4290 \\ 160 & 4302.939 & -6.131 & -3.766 & 4292 \\ 160.2 & 4304.743 & -6.186 & -3.032 & 4293 \\ 160.4 & 4306.53 & -6.339 & -3.29 & 4294 \\ 160.6 & 4308.298 & -6.595 & -3.762 & 4296 \\ 160.8 & 4310.048 & -5.982 & -3.598 & 4297 \\ 161.2 & 4313.493 & -6.164 & -4.245 & 4300 \\ 161.4 & 4315.189 & -6.672 & -3.988 & 4301 \\ 161.6 & 4316.888 & -6.834 & -3.843 & 4303 \\ 161.8 & 4318.715 & -6.953 & -2.985 & 4305 \\ 162 & 4320.682 & -7.004 & -2.983 & 4307\end{array}$




$\begin{array}{ccccc}162.2 & 4322.779 & -7.249 & -3.352 & 4309 \\ 162.4 & 4324.998 & -7.111 & -3.985 & 4311 \\ 162.6 & 4327.328 & -6.842 & -3.604 & 4313 \\ 162.8 & 4329.761 & -6.886 & -3.599 & 4315 \\ 163 & 4332.287 & -6.676 & -3.584 & 4317 \\ 163.2 & 4334.896 & -7.04 & -4.509 & 4319 \\ 163.4 & 4337.58 & -7.306 & -4.357 & 4321 \\ 163.6 & 4340.329 & -7.225 & -3.726 & 4323 \\ 163.8 & 4343.133 & -6.979 & -3.421 & 4325 \\ 164 & 4345.983 & -6.964 & -3.729 & 4327 \\ 164.2 & 4348.87 & -7.013 & -3.457 & 4329 \\ 164.4 & 4351.784 & -7.552 & -4.019 & 4331 \\ 164.6 & 4354.716 & -7.454 & -4.397 & 4333 \\ 164.8 & 4357.657 & -7.398 & -4.065 & 4335 \\ 165 & 4360.597 & -7.347 & -3.345 & 4337 \\ 165.2 & 4363.527 & -7.288 & -2.971 & 4339 \\ 165.4 & 4366.437 & -6.925 & -3.829 & 4341 \\ 165.6 & 4369.318 & -7.161 & -3.663 & 4343 \\ 165.8 & 4372.161 & -6.952 & -3.615 & 4345 \\ 166 & 4374.956 & -6.825 & -3.73 & 4347 \\ 166.2 & 4377.694 & -6.633 & -3.422 & 4349 \\ 166.4 & 4380.365 & -6.727 & -4.334 & 4351 \\ 166.6 & 4382.961 & -6.755 & -4.395 & 4353 \\ 166.8 & 4385.471 & -6.514 & -3.348 & 4355 \\ 167 & 4387.886 & -6.61 & -3.325 & 4357 \\ 167.2 & 4390.198 & -6.595 & -3.532 & 4359 \\ 167.4 & 4392.396 & -6.698 & -3.218 & 4361 \\ 167.6 & 4394.471 & -6.561 & -3.822 & 4363 \\ 167.8 & 4396.414 & -6.832 & -4.351 & 4365 \\ 168 & 4398.216 & -6.878 & -2.948 & 4367 \\ 168.2 & 4399.867 & -6.949 & -3.041 & 4369 \\ 168.4 & 4401.357 & -7.214 & -3.452 & 4371 \\ 168.6 & 4402.695 & -7.505 & -3.456 & 4373 \\ 168.8 & 4403.987 & -7.337 & -3.443 & 4373 \\ 169 & 4405.254 & -7.382 & -3.343 & 4373 \\ 169.2 & 4406.501 & -7.193 & -2.674 & 4373 \\ 169.4 & 4407.733 & -7.519 & -2.93 & 4373 \\ 169.6 & 4408.953 & -7.18 & -2.997 & 4373 \\ 169.8 & 4410.166 & -7.494 & -3.48 & 4373 \\ 170 & 4411.377 & -7.449 & -3.511 & 4373 \\ 170.2 & 4412.59 & -7.057 & -3.605 & 4373 \\ 170.4 & 4413.81 & -6.647 & -3.433 & 4373 \\ 170.6 & 4415.042 & -5.668 & -3.791 & 4373 \\ 170.8 & 4416.289 & -6.49 & -4.231 & 4373 \\ 171 & 4417.556 & -6.937 & -4.403 & 4373 \\ 171.2 & 4418.848 & -6.859 & -4.794 & 4373 \\ 171.4 & 4420.169 & -7.341 & -4.295 & 4373 \\ 171.6 & 4421.524 & -7.113 & -3.921 & 4373 \\ 171.8 & 4422.917 & -7.188 & -4.345 & 4373 \\ 172.2 & 4424.353 & -7.065 & -4.039 & 4373 \\ & 4425.836 & -7.225 & -4.145 & 4374\end{array}$




$\begin{array}{ccccc}172.4 & 4427.371 & -7.156 & -3.574 & 4374 \\ 172.6 & 4428.962 & -6.869 & -2.844 & 4374 \\ 172.8 & 4430.613 & -6.933 & -3.305 & 4374 \\ 173 & 4432.329 & -6.89 & -3.61 & 4374 \\ 173.2 & 4434.116 & -7.216 & -4.202 & 4374 \\ 173.4 & 4435.976 & -7.143 & -4.035 & 4374 \\ 173.6 & 4438.011 & -7.077 & -3.739 & 4378 \\ 173.8 & 4440.774 & -6.951 & -3.953 & 4388 \\ 174 & 4444.302 & -6.839 & -3.563 & 4397 \\ 174.2 & 4448.533 & -6.836 & -3.597 & 4406 \\ 174.4 & 4453.405 & -6.694 & -3.675 & 4415 \\ 174.6 & 4458.855 & -6.736 & -3.873 & 4425 \\ 174.8 & 4464.82 & -6.733 & -3.9 & 4434 \\ 175 & 4471.24 & -6.777 & -4.234 & 4443 \\ 175.2 & 4478.051 & -6.64 & -4.384 & 4452 \\ 175.4 & 4485.191 & -7.06 & -4.636 & 4461 \\ 175.6 & 4492.598 & -7.154 & -4.444 & 4471 \\ 175.8 & 4500.21 & -6.852 & -3.664 & 4480 \\ 176 & 4507.964 & -6.877 & -3.695 & 4489 \\ 176.2 & 4515.799 & -6.49 & -3.048 & 4498 \\ 176.4 & 4523.652 & -6.289 & -3.535 & 4507 \\ 176.6 & 4531.46 & -6.397 & -3.877 & 4517 \\ 176.8 & 4539.162 & -6.415 & -4.198 & 4526 \\ 177 & 4546.695 & -6.502 & -4.054 & 4535 \\ 177.2 & 4553.997 & -7.118 & -3.693 & 4544 \\ 177.4 & 4561.005 & -7.175 & -3.923 & 4554 \\ 177.6 & 4567.659 & -7.034 & -4.295 & 4563 \\ 177.8 & 4573.894 & -7.044 & -4.686 & 4572 \\ 178 & 4579.649 & -7.086 & -3.813 & 4581 \\ 178.2 & 4584.862 & -6.949 & -3.45 & 4590 \\ 178.4 & 4589.471 & -6.734 & -3.384 & 4600 \\ 178.6 & 4593.515 & -6.612 & -3.489 & 4606 \\ 178.8 & 4597.564 & -6.839 & -3.45 & 4611 \\ 179 & 4601.705 & -6.81 & -3.503 & 4615 \\ 179.2 & 4605.924 & -5.982 & -3.712 & 4620 \\ 179.4 & 4610.207 & -4.696 & -3.643 & 4624 \\ 179.6 & 4614.542 & -4.027 & -3.4 & 4629 \\ 179.8 & 4618.915 & -3.774 & -2.694 & 4633 \\ 180 & 4623.312 & -3.875 & -3.133 & 4638 \\ 180.2 & 4627.72 & -4.068 & -3.034 & 4642 \\ 180.4 & 4632.125 & -4.556 & -2.555 & 4647 \\ 180.6 & 4636.514 & -4.982 & -2.449 & 4651 \\ 180.8 & 4640.873 & -4.65 & -2.347 & 4656 \\ 181 & 4645.189 & -4.444 & -2.463 & 4660 \\ 181.2 & 4649.449 & -5.064 & -2.414 & 4665 \\ 181.4 & 4653.638 & -5.161 & -3.161 & 4669 \\ 181.6 & 4657.743 & -5.057 & -3.401 & 4674 \\ 181.8 & 4661.752 & -5.072 & -2.574 & 4678 \\ 182 & 4665.65 & -5.386 & -2.966 & 4683 \\ 182.2 & 4669.424 & -5.6 & -2.906 & 4687 \\ 182.4 & 4673.06 & -5.48 & -3.39 & 4692\end{array}$




$\begin{array}{ccccc}182.6 & 4676.545 & -5.435 & -4.323 & 4696 \\ 182.8 & 4679.866 & -5.352 & -4.494 & 4701 \\ 183 & 4683.008 & -5.136 & -4.415 & 4705 \\ 183.2 & 4685.959 & -4.872 & -3.931 & 4710 \\ 183.4 & 4688.705 & -4.746 & -4.241 & 4714 \\ 183.6 & 4691.233 & -5.005 & -3.653 & 4719 \\ 183.8 & 4693.528 & -5.242 & -2.818 & 4723 \\ 184 & 4695.578 & -5.157 & -2.592 & 4728 \\ 184.2 & 4697.494 & -5.3 & -2.572 & 4729 \\ 184.4 & 4699.396 & -5.406 & -2.967 & 4730 \\ 184.6 & 4701.285 & -5.396 & -3.348 & 4732 \\ 184.8 & 4703.161 & -5.342 & -2.795 & 4733 \\ 185 & 4705.024 & -5.067 & -3.316 & 4734 \\ 185.2 & 4706.876 & -5.273 & -3.539 & 4736 \\ 185.4 & 4708.715 & -5.665 & -2.694 & 4737 \\ 185.6 & 4710.542 & -5.096 & -2.975 & 4738 \\ 185.8 & 4712.359 & -5.584 & -2.559 & 4740 \\ 186 & 4714.164 & -5.332 & -2.716 & 4741 \\ 186.2 & 4715.959 & -5.098 & -2.879 & 4742 \\ 186.4 & 4717.743 & -5.237 & -3.311 & 4744 \\ 186.6 & 4719.518 & -5.4 & -3.688 & 4745 \\ 186.8 & 4721.282 & -5.282 & -3.21 & 4746 \\ 187 & 4723.038 & -5.56 & -3.216 & 4748 \\ 187.2 & 4724.784 & -5.715 & -4.141 & 4749 \\ 187.4 & 4726.521 & -5.885 & -3.602 & 4750 \\ 187.6 & 4728.25 & -6.353 & -3.604 & 4752 \\ 187.8 & 4729.971 & -6.333 & -3.702 & 4753 \\ 188 & 4731.685 & -6.339 & -3.616 & 4754 \\ 188.2 & 4733.391 & -6.132 & -3.079 & 4755 \\ 188.4 & 4735.089 & -6.27 & -2.504 & 4757 \\ 188.6 & 4736.781 & -5.323 & -1.957 & 4758 \\ 188.8 & 4738.467 & -5.251 & -2.186 & 4759 \\ 189 & 4740.146 & -5.413 & -2.263 & 4761 \\ 189.2 & 4741.819 & -5.943 & -2.602 & 4762 \\ 189.4 & 4743.487 & -5.84 & -3.025 & 4763 \\ 189.6 & 4745.15 & -5.308 & -3.107 & 4765 \\ 189.8 & 4746.808 & -5.796 & -2.941 & 4766 \\ 190 & 4748.462 & -5.896 & -2.758 & 4767 \\ 190.2 & 4750.111 & -6.14 & -3.067 & 4769 \\ 190.4 & 4751.756 & -6.162 & -2.994 & 4770 \\ 190.6 & 4753.398 & -6.055 & -3.248 & 4771 \\ 190.8 & 4755.036 & -6.125 & -3.875 & 4773 \\ 191 & 4756.672 & -6.295 & -4.023 & 4774 \\ 191.2 & 4758.305 & -6.288 & -3.703 & 4775 \\ 191.4 & 4759.935 & -6.208 & -3.39 & 4777 \\ 191.6 & 4761.564 & -6.492 & -3.351 & 4778 \\ 191.8 & 4763.191 & -6.218 & -3.477 & 4779 \\ 192 & 4764.817 & -6.568 & -3.991 & 4781 \\ 192.2 & 4766.442 & -6.689 & -3.701 & 4782 \\ 192.4 & 4768.066 & -6.699 & -4.064 & 4783 \\ 192.6 & 4769.69 & -7.138 & -5.007 & 4785\end{array}$




$\begin{array}{ccccc}192.8 & 4771.313 & -6.455 & -5.014 & 4786 \\ 193 & 4772.937 & -6.202 & -4.537 & 4787 \\ 193.2 & 4774.562 & -6.648 & -4.102 & 4789 \\ 193.4 & 4776.188 & -6.001 & -3.395 & 4790 \\ 193.6 & 4777.815 & -6.125 & -3.761 & 4791 \\ 193.8 & 4779.443 & -6.497 & -4.016 & 4793 \\ 194 & 4781.073 & -6.804 & -4.676 & 4794 \\ 194.2 & 4782.706 & -6.299 & -4.106 & 4795 \\ 194.4 & 4784.341 & -6.026 & -4.534 & 4797 \\ 194.6 & 4785.979 & -5.932 & -4.143 & 4798 \\ 194.8 & 4787.62 & -6.322 & -4.07 & 4799 \\ 195 & 4789.265 & -6.424 & -3.928 & 4801 \\ 195.2 & 4790.914 & -6.399 & -4.096 & 4802 \\ 195.4 & 4792.566 & -6.592 & -4.65 & 4803 \\ 195.6 & 4794.224 & -6.638 & -4.103 & 4805 \\ 195.8 & 4795.886 & -6.425 & -3.828 & 4806 \\ 196 & 4797.553 & -6.773 & -4.342 & 4807 \\ 196.2 & 4799.226 & -6.67 & -4.056 & 4809 \\ 196.4 & 4800.904 & -6.688 & -3.731 & 4810 \\ 196.6 & 4802.588 & -6.677 & -3.454 & 4811 \\ 196.8 & 4804.279 & -6.801 & -4.357 & 4813 \\ 197 & 4805.977 & -7.047 & -4.373 & 4814 \\ 197.2 & 4807.682 & -6.948 & -3.712 & 4815 \\ 197.4 & 4809.394 & -6.309 & -3.951 & 4817 \\ 197.6 & 4811.114 & -6.43 & -3.666 & 4818 \\ 197.8 & 4812.842 & -6.351 & -3.146 & 4819 \\ 198 & 4814.578 & -6.493 & -3.656 & 4820 \\ 198.2 & 4816.323 & -6.487 & -4.408 & 4822 \\ 198.4 & 4818.077 & -6.9 & -5.134 & 4823 \\ 198.6 & 4819.84 & -7.019 & -4.666 & 4824 \\ 198.8 & 4821.613 & -6.596 & -4.336 & 4826 \\ 199 & 4823.396 & -6.754 & -4.479 & 4827 \\ 199.2 & 4825.189 & -6.442 & -4.821 & 4828 \\ 199.4 & 4826.993 & -6.707 & -4.105 & 4830 \\ 199.6 & 4828.807 & -6.499 & -4.17 & 4831 \\ 199.8 & 4830.633 & -6.66 & -4.507 & 4832 \\ 200 & 4832.471 & -6.802 & -4.283 & 4834 \\ 200.2 & 4834.32 & -6.665 & -3.937 & 4835 \\ 200.4 & 4836.182 & -6.637 & -4.674 & 4836 \\ 200.6 & 4838.056 & -6.983 & -3.896 & 4838 \\ 200.8 & 4839.943 & -6.921 & -3.874 & 4839 \\ 201 & 4841.843 & -6.874 & -4.051 & 4840 \\ 201.2 & 4843.757 & -7.439 & -4.348 & 4842 \\ 201.4 & 4845.685 & -7.684 & -4.251 & 4843 \\ 201.6 & 4847.626 & -7.347 & -4.061 & 4844 \\ 201.8 & 4849.583 & -7.378 & -3.675 & 4846 \\ 202 & 4851.554 & -7.583 & -3.772 & 4847 \\ 202.2 & 4853.54 & -7.712 & -3.226 & 4848 \\ 202.4 & 4855.541 & -7.705 & -3.489 & 4850 \\ 202.6 & 4857.565 & -7.815 & -4.192 & 4852 \\ 202.8 & 4859.648 & -7.893 & -4.564 & 4855\end{array}$




$\begin{array}{ccccc}203 & 4861.795 & -8.334 & -4.087 & 4859 \\ 203.2 & 4864.006 & -8.313 & -3.567 & 4862 \\ 203.4 & 4866.278 & -7.599 & -3.45 & 4866 \\ 203.6 & 4868.611 & -6.874 & -2.873 & 4869 \\ 203.8 & 4871.002 & -6.943 & -2.986 & 4873 \\ 204 & 4873.452 & -6.485 & -3.523 & 4876 \\ 204.2 & 4875.958 & -5.764 & -3.818 & 4879 \\ 204.4 & 4878.52 & -6.358 & -3.527 & 4883 \\ 204.6 & 4881.135 & -6.547 & -3.872 & 4886 \\ 204.8 & 4883.803 & -6.686 & -3.914 & 4890 \\ 205 & 4886.522 & -6.604 & -3.851 & 4893 \\ 205.2 & 4889.291 & -6.532 & -3.349 & 4897 \\ 205.4 & 4892.109 & -6.62 & -3.26 & 4900 \\ 205.6 & 4894.974 & -6.526 & -3.055 & 4904 \\ 205.8 & 4897.885 & -6.196 & -2.291 & 4907 \\ 206 & 4900.841 & -6.179 & -2.143 & 4910 \\ 206.2 & 4903.84 & -5.957 & -2.286 & 4914 \\ 206.4 & 4906.881 & -6.133 & -2.067 & 4917 \\ 206.6 & 4909.963 & -6.509 & -2.331 & 4921 \\ 206.8 & 4913.085 & -6.839 & -2.372 & 4924 \\ 207 & 4916.245 & -7.012 & -2.71 & 4928 \\ 207.2 & 4919.441 & -7.106 & -2.884 & 4931 \\ 207.4 & 4922.673 & -6.779 & -2.911 & 4934 \\ 207.6 & 4925.94 & -6.969 & -3.357 & 4938 \\ 207.8 & 4929.239 & -6.876 & -3.65 & 4941 \\ 208 & 4932.57 & -6.45 & -3.688 & 4945 \\ 208.2 & 4935.931 & -6.344 & -3.62 & 4948 \\ 208.4 & 4939.321 & -6.067 & -3.969 & 4952 \\ 208.6 & 4942.738 & -6.487 & -3.918 & 4955 \\ 208.8 & 4946.182 & -6.283 & -3.725 & 4958 \\ 209 & 4949.651 & -6.312 & -4.183 & 4962 \\ 209.2 & 4953.144 & -6.035 & -4.236 & 4965 \\ 209.4 & 4956.659 & -5.762 & -3.595 & 4969 \\ 209.6 & 4960.196 & -6.053 & -3.317 & 4972 \\ 209.8 & 4963.752 & -5.927 & -3.486 & 4976 \\ 210 & 4967.327 & -5.85 & -3.671 & 4979 \\ 210.2 & 4970.918 & -5.844 & -3.995 & 4982 \\ 210.4 & 4974.526 & -5.943 & -3.823 & 4986 \\ 210.6 & 4978.148 & -6.255 & -3.463 & 4989 \\ 210.8 & 4981.783 & -6.311 & -3.127 & 4993 \\ 211 & 4985.43 & -6.231 & -3.153 & 4996 \\ 211.2 & 4989.088 & -6.057 & -3.203 & 5000 \\ 211.4 & 4992.755 & -6.01 & -3.296 & 5003 \\ 211.6 & 4996.431 & -6.026 & -3.715 & 5006 \\ 211.8 & 5000.112 & -6.446 & -3.826 & 5010 \\ 212 & 5003.8 & -6.316 & -3.763 & 5013 \\ 212.2 & 5007.491 & -6.62 & -3.815 & 5017 \\ 212.4 & 5011.185 & -6.925 & -3.154 & 5020 \\ 212.6 & 5014.88 & -7.15 & -3.195 & 5024 \\ 212.8 & 5018.575 & -6.892 & -3.208 & 5027 \\ 213 & 5022.27 & -6.928 & -3.781 & 5030\end{array}$




$\begin{array}{ccccc}213.2 & 5025.961 & -7 & -3.868 & 5034 \\ 213.4 & 5029.649 & -7.091 & -3.832 & 5037 \\ 213.6 & 5033.332 & -7.123 & -4.424 & 5041 \\ 213.8 & 5037.008 & -7.214 & -4.93 & 5044 \\ 214 & 5040.677 & -7.42 & -4.828 & 5048 \\ 214.2 & 5044.336 & -6.982 & -4.407 & 5051 \\ 214.4 & 5047.985 & -7.023 & -4.713 & 5054 \\ 214.6 & 5051.623 & -7.06 & -4.01 & 5058 \\ 214.8 & 5055.247 & -6.599 & -3.55 & 5061 \\ 215 & 5058.857 & -6.819 & -3.591 & 5065 \\ 215.2 & 5062.451 & -7.084 & -3.564 & 5068 \\ 215.4 & 5066.029 & -7.08 & -4.094 & 5072 \\ 215.6 & 5069.588 & -7.238 & -5.019 & 5075 \\ 215.8 & 5073.127 & -7.313 & -5.428 & 5078 \\ 216 & 5076.646 & -7.187 & -4.217 & 5082 \\ 216.2 & 5080.143 & -7.135 & -4.405 & 5085 \\ 216.4 & 5083.616 & -7.138 & -4.341 & 5089 \\ 216.6 & 5087.064 & -6.908 & -3.914 & 5092 \\ 216.8 & 5090.486 & -6.741 & -4.451 & 5096 \\ 217 & 5093.88 & -6.97 & -4.148 & 5099 \\ 217.2 & 5097.246 & -7.136 & -4.141 & 5102 \\ 217.4 & 5100.582 & -6.896 & -4.439 & 5106 \\ 217.6 & 5103.886 & -7.031 & -4.572 & 5109 \\ 217.8 & 5107.158 & -7.269 & -4.282 & 5113 \\ 218 & 5110.396 & -7.431 & -4.343 & 5116 \\ 218.2 & 5113.598 & -7.34 & -5.095 & 5120 \\ 218.4 & 5116.764 & -7.246 & -5.622 & 5123 \\ 218.6 & 5119.892 & -7.359 & -5.666 & 5127 \\ 218.8 & 5122.98 & -7.207 & -4.577 & 5130 \\ 219 & 5126.028 & -6.977 & -4.374 & 5133 \\ 219.2 & 5129.034 & -6.769 & -3.368 & 5137 \\ 219.4 & 5131.997 & -6.537 & -2.707 & 5140 \\ 219.6 & 5134.931 & -6.997 & -3.56 & 5143 \\ 219.8 & 5137.922 & -6.99 & -3.002 & 5145 \\ 220 & 5140.983 & -6.96 & -3.263 & 5146 \\ 220.2 & 5144.108 & -6.576 & -3.431 & 5148 \\ 220.4 & 5147.296 & -6.703 & -3.193 & 5150 \\ 220.6 & 5150.541 & -6.584 & -3.101 & 5152 \\ 220.8 & 5153.84 & -6.394 & -3.401 & 5153 \\ 221 & 5157.189 & -6.521 & -3.541 & 5155 \\ 221.2 & 5160.585 & -6.499 & -3.596 & 5157 \\ 221.4 & 5164.022 & -6.266 & -3.622 & 5159 \\ 221.6 & 5167.499 & -6.323 & -3.586 & 5160 \\ 221.8 & 5171.01 & -6.631 & -3.131 & 5162 \\ 222 & 5174.552 & -6.47 & -3.111 & 5164 \\ 222.2 & 5178.122 & -6.411 & -3.454 & 5166 \\ 222.4 & 5181.715 & -6.679 & -3.518 & 5168 \\ 222.6 & 5185.327 & -6.506 & -2.552 & 5169 \\ 222.8 & 5188.955 & -6.61 & -2.9 & 5171 \\ 23.2 & 5192.595 & -6.841 & -3.218 & 5173 \\ & 5196.243 & -7.238 & -3.422 & 5175\end{array}$




$\begin{array}{ccccc}223.4 & 5199.895 & -6.783 & -2.807 & 5176 \\ 223.6 & 5203.548 & -7.009 & -3.419 & 5178 \\ 223.8 & 5207.198 & -6.593 & -3.497 & 5180 \\ 224 & 5210.84 & -6.667 & -3.509 & 5182 \\ 224.2 & 5214.471 & -6.766 & -3.509 & 5183 \\ 224.4 & 5218.087 & -6.608 & -3.32 & 5185 \\ 224.6 & 5221.685 & -6.473 & -2.957 & 5187 \\ 224.8 & 5225.26 & -6.698 & -3.429 & 5189 \\ 225 & 5228.809 & -6.812 & -2.72 & 5190 \\ 225.2 & 5232.328 & -6.931 & -3.028 & 5192 \\ 225.4 & 5235.812 & -7.177 & -3.336 & 5194 \\ 225.6 & 5239.259 & -6.988 & -2.997 & 5196 \\ 225.8 & 5242.665 & -6.865 & -2.948 & 5198 \\ 226 & 5246.025 & -7.45 & -3.41 & 5199 \\ 226.2 & 5249.336 & -7.731 & -3.594 & 5201 \\ 226.4 & 5252.594 & -7.59 & -3.614 & 5203 \\ 226.6 & 5255.795 & -7.591 & -3.754 & 5205 \\ 226.8 & 5258.935 & -7.656 & -3.823 & 5206 \\ 227 & 5262.011 & -7.37 & -3.739 & 5208 \\ 227.2 & 5265.018 & -7.313 & -3.851 & 5210 \\ 227.4 & 5267.954 & -6.964 & -3.382 & 5212 \\ 227.6 & 5270.84 & -6.788 & -3.05 & 5215 \\ 227.8 & 5273.837 & -6.45 & -3.123 & 5221 \\ 228 & 5276.965 & -6.046 & -3.531 & 5227 \\ 228.2 & 5280.22 & -5.664 & -2.528 & 5232 \\ 228.4 & 5283.594 & -6.343 & -2.848 & 5238 \\ 228.6 & 5287.084 & -6.809 & -3.207 & 5244 \\ 228.8 & 5290.683 & -6.681 & -3.356 & 5249 \\ 229 & 5294.385 & -6.213 & -3.412 & 5255 \\ 229.2 & 5298.186 & -6.35 & -4.074 & 5260 \\ 229.4 & 5302.078 & -6.462 & -3.583 & 5266 \\ 229.6 & 5306.058 & -6.72 & -3.933 & 5272 \\ 229.8 & 5310.119 & -6.471 & -4.59 & 5277 \\ 230 & 5314.255 & -6.601 & -4.357 & 5283 \\ 230.2 & 5318.462 & -6.858 & -4.05 & 5289 \\ 230.4 & 5322.733 & -7.253 & -4.009 & 5294 \\ 230.6 & 5327.062 & -7.048 & -3.439 & 5300 \\ 230.8 & 5331.445 & -7.023 & -3.407 & 5306 \\ 231 & 5335.875 & -6.958 & -3.968 & 5311 \\ 231.2 & 5340.348 & -7.085 & -4.388 & 5317 \\ 231.4 & 5344.856 & -6.867 & -3.768 & 5322 \\ 231.6 & 5349.396 & -6.765 & -3.225 & 5328 \\ 231.8 & 5353.96 & -6.662 & -3.253 & 5334 \\ 232 & 5358.544 & -6.801 & -3.179 & 5339 \\ 232.2 & 5363.142 & -7.007 & -2.914 & 5345 \\ 232.4 & 5367.749 & -6.846 & -3.087 & 5351 \\ 232.6 & 5372.358 & -7.023 & -3.238 & 5356 \\ 232.8 & 5376.964 & -6.724 & -3.054 & 5362 \\ 233.2 & 5381.561 & -6.628 & -2.946 & 5368 \\ & 5386.145 & -6.353 & -2.873 & 5373 \\ 233 & 5390.709 & -6.626 & -3.092 & 5379\end{array}$




\begin{tabular}{|c|c|c|c|c|}
\hline 233.6 & 5395.248 & -6.518 & -3.27 & 5385 \\
\hline 233.8 & 5399.755 & -6.469 & -3.273 & 5390 \\
\hline 234 & 5404.226 & -5.778 & -2.962 & 5396 \\
\hline 234.2 & 5408.655 & -5.016 & -2.703 & 5401 \\
\hline 234.4 & 5413.037 & -5.03 & -2.638 & 5407 \\
\hline 234.6 & 5417.364 & -4.485 & -2.138 & 5413 \\
\hline 234.8 & 5421.633 & -5.126 & -2.156 & 5418 \\
\hline 235 & 5425.838 & -5.628 & -2.809 & 5424 \\
\hline 235.2 & 5429.972 & -5.475 & -3.164 & 5430 \\
\hline 235.4 & 5434.03 & -4.257 & -2.428 & 5435 \\
\hline 235.6 & 5438.008 & -5.815 & -3.559 & 5441 \\
\hline 235.8 & 5441.898 & -6.452 & -3.413 & 5447 \\
\hline 236 & 5445.695 & -6.277 & -3.469 & 5452 \\
\hline 236.2 & 5449.395 & -6.269 & -4.036 & 5458 \\
\hline 236.4 & 5452.99 & -6.708 & -4.465 & 5463 \\
\hline 236.6 & 5456.477 & -6.748 & -3.258 & 5469 \\
\hline 236.8 & 5459.848 & -5.545 & -2.848 & 5475 \\
\hline 237 & 5463.098 & -6.836 & -3.785 & 5480 \\
\hline 237.2 & 5466.223 & -7.136 & -4.237 & 5486 \\
\hline 237.4 & 5469.215 & -7.061 & -3.842 & 5492 \\
\hline 237.6 & 5472.07 & -6.677 & -3.695 & 5497 \\
\hline 237.8 & 5474.783 & -5.213 & -3.302 & 5503 \\
\hline 238 & 5477.346 & -3.791 & -1.699 & 5509 \\
\hline 238.2 & 5479.843 & -4.699 & -2.047 & 5510 \\
\hline 238.4 & 5482.36 & -6.556 & -2.904 & 5510 \\
\hline 238.6 & 5484.893 & -6.944 & -3.066 & 5511 \\
\hline 238.8 & 5487.442 & -7.187 & -2.923 & 5512 \\
\hline 239 & 5490.005 & -6.471 & -2.659 & 5513 \\
\hline 239.2 & 5492.58 & -6.408 & -2.763 & 5514 \\
\hline 239.4 & 5495.165 & -6.64 & -2.898 & 5515 \\
\hline 239.6 & 5497.759 & -6.833 & -3.049 & 5516 \\
\hline 239.8 & 5500.359 & -6.697 & -3.116 & 5517 \\
\hline 240 & 5502.965 & -6.555 & -3.162 & 5518 \\
\hline 240.2 & 5505.574 & -6.302 & -3.194 & 5519 \\
\hline 240.4 & 5508.185 & -6.6 & -3.339 & 5520 \\
\hline 240.6 & 5510.796 & -6.871 & -3.125 & 5521 \\
\hline 240.8 & 5513.405 & -6.988 & -3.212 & 5522 \\
\hline 241 & 5516.01 & -7.104 & -3.457 & 5523 \\
\hline 241.2 & 5518.611 & -7.006 & -3.284 & 5524 \\
\hline 241.4 & 5521.204 & -6.751 & -3.304 & 5524 \\
\hline 241.6 & 5523.788 & -6.857 & -2.949 & 5525 \\
\hline 241.8 & 5526.362 & -6.857 & -2.864 & 5526 \\
\hline 242 & 5528.924 & -6.768 & -2.882 & 5527 \\
\hline 242.2 & 5531.472 & -6.889 & -3.021 & 5528 \\
\hline 242.4 & 5534.005 & -6.796 & -3.016 & 5529 \\
\hline 242.6 & 5536.52 & -6.964 & -3.008 & 5530 \\
\hline 242.8 & 5539.016 & -7.106 & -3.335 & 5531 \\
\hline 243 & 5541.491 & -7.177 & -3.464 & 5532 \\
\hline 243.2 & 5543.944 & -7.436 & -3.361 & 5533 \\
\hline 243.4 & 5546.372 & -7.606 & -3.53 & 5534 \\
\hline 243.6 & 5548.775 & -7.607 & -3.828 & 5535 \\
\hline
\end{tabular}




\begin{tabular}{|c|c|c|c|c|}
\hline 243.8 & 5551.149 & -7.604 & -3.87 & 5536 \\
\hline 244 & 5553.495 & -7.402 & -3.898 & 5537 \\
\hline 244.2 & 5555.809 & -6.841 & -4.029 & 5538 \\
\hline 244.4 & 5558.09 & -6.324 & -3.526 & 5538 \\
\hline 244.6 & 5560.336 & -5.515 & -3.126 & 5539 \\
\hline 244.8 & 5562.546 & -6.271 & -3.687 & 5540 \\
\hline 245 & 5564.718 & -6.578 & -4.283 & 5541 \\
\hline 245.2 & 5566.851 & -6.089 & -3.658 & 5542 \\
\hline 245.4 & 5568.941 & -6.791 & -4.574 & 5543 \\
\hline 245.6 & 5570.996 & -6.672 & -3.78 & 5545 \\
\hline 245.8 & 5573.051 & -6.002 & -3.739 & 5547 \\
\hline 246 & 5575.116 & -6.094 & -3.843 & 5550 \\
\hline 246.2 & 5577.189 & -5.885 & -3.435 & 5552 \\
\hline 246.4 & 5579.271 & -5.772 & -2.588 & 5555 \\
\hline 246.6 & 5581.362 & -6.207 & -3.259 & 5557 \\
\hline 246.8 & 5583.461 & -6.319 & -3.439 & 5560 \\
\hline 247 & 5585.57 & -6.491 & -3.656 & 5562 \\
\hline 247.2 & 5587.687 & -6.36 & -3.121 & 5565 \\
\hline 247.4 & 5589.813 & -6.469 & -3.391 & 5567 \\
\hline 247.6 & 5591.948 & -6.073 & -3.715 & 5570 \\
\hline 247.8 & 5594.092 & -6.322 & -3.403 & 5572 \\
\hline 248 & 5596.244 & -6.887 & -3.831 & 5575 \\
\hline 248.2 & 5598.407 & -6.223 & -2.875 & 5577 \\
\hline 248.4 & 5600.578 & -6.397 & -3.386 & 5580 \\
\hline 248.6 & 5602.758 & -6.152 & -3.407 & 5582 \\
\hline 248.8 & 5604.948 & -5.776 & -2.606 & 5585 \\
\hline 249 & 5607.146 & -6.243 & -2.569 & 5587 \\
\hline 249.2 & 5609.354 & -6.799 & -3.136 & 5590 \\
\hline 249.4 & 5611.572 & -6.71 & -3.308 & 5592 \\
\hline 249.6 & 5613.799 & -7.25 & -3.374 & 5595 \\
\hline 249.8 & 5616.035 & -7.28 & -3.724 & 5597 \\
\hline 250 & 5618.28 & -7.184 & -3.953 & 5599 \\
\hline 250.2 & 5620.536 & -6.995 & -3.511 & 5602 \\
\hline 250.4 & 5622.801 & -7.212 & -4.863 & 5604 \\
\hline 250.6 & 5625.075 & -7.012 & -3.409 & 5607 \\
\hline 250.8 & 5627.359 & -6.875 & -3.593 & 5609 \\
\hline 251 & 5629.653 & -6.457 & -3.908 & 5612 \\
\hline 251.2 & 5631.956 & -7.341 & -3.821 & 5614 \\
\hline 251.4 & 5634.27 & -7.646 & -4.039 & 5617 \\
\hline 251.6 & 5636.593 & -7.681 & -3.874 & 5619 \\
\hline 251.8 & 5638.926 & -7.51 & -3.717 & 5622 \\
\hline 252 & 5641.268 & -7.567 & -3.835 & 5624 \\
\hline 252.2 & 5643.621 & -7.744 & -3.886 & 5627 \\
\hline 252.4 & 5645.984 & -7.791 & -4.061 & 5629 \\
\hline 252.6 & 5648.357 & -7.654 & -4.001 & 5632 \\
\hline 252.8 & 5650.74 & -7.81 & -4.373 & 5634 \\
\hline 253 & 5653.133 & -7.758 & -4.537 & 5637 \\
\hline 253.2 & 5655.537 & -7.9 & -4.56 & 5639 \\
\hline 253.4 & 5657.95 & -7.934 & -3.85 & 5642 \\
\hline 253.6 & 5660.374 & -7.537 & -4.362 & 5644 \\
\hline 253.8 & 5662.808 & -7.646 & -5.113 & 5647 \\
\hline
\end{tabular}




$\begin{array}{ccccc}254 & 5665.252 & -7.642 & -4.867 & 5649 \\ 254.2 & 5667.707 & -7.62 & -4.925 & 5652 \\ 254.4 & 5670.173 & -8.039 & -4.319 & 5654 \\ 254.6 & 5672.649 & -7.735 & -3.902 & 5657 \\ 254.8 & 5675.135 & -7.638 & -4.059 & 5659 \\ 255 & 5677.632 & -7.36 & -3.922 & 5662 \\ 255.2 & 5680.139 & -7.056 & -4.179 & 5664 \\ 255.4 & 5682.658 & -7.056 & -4.06 & 5667 \\ 255.6 & 5685.186 & -6.526 & -3.868 & 5669 \\ 255.8 & 5687.726 & -6.898 & -4.833 & 5672 \\ 256 & 5690.277 & -6.863 & -4.247 & 5674 \\ 256.2 & 5692.838 & -5.972 & -3.577 & 5677 \\ 256.4 & 5695.41 & -6.432 & -3.544 & 5679 \\ 256.6 & 5697.993 & -7.113 & -4.195 & 5682 \\ 256.8 & 5700.588 & -7.09 & -4.51 & 5684 \\ 257 & 5703.193 & -6.805 & -4.005 & 5686 \\ 257.2 & 5705.809 & -6.681 & -3.763 & 5689 \\ 257.4 & 5708.436 & -6.857 & -3.367 & 5691 \\ 257.6 & 5711.075 & -6.679 & -3.699 & 5694 \\ 257.8 & 5713.725 & -6.664 & -3.604 & 5696 \\ 258 & 5716.385 & -6.555 & -2.752 & 5699 \\ 258.2 & 5719.058 & -6.603 & -2.837 & 5701 \\ 258.4 & 5721.741 & -6.917 & -3.215 & 5704 \\ 258.6 & 5724.436 & -7.183 & -3.27 & 5706 \\ 258.8 & 5727.142 & -6.868 & -2.917 & 5709 \\ 259 & 5729.86 & -7.201 & -3.751 & 5711 \\ 259.2 & 5732.59 & -7.048 & -4.133 & 5714 \\ 259.4 & 5735.33 & -7.143 & -3.255 & 5716 \\ 259.6 & 5738.083 & -6.776 & -2.683 & 5719 \\ 259.8 & 5740.847 & -7.027 & -3.34 & 5721 \\ 260 & 5743.623 & -6.876 & -3.276 & 5724 \\ 260.2 & 5746.453 & -6.736 & -3.204 & 5728 \\ 260.4 & 5749.38 & -6.914 & -3.464 & 5733 \\ 260.6 & 5752.399 & -6.783 & -3.225 & 5737 \\ 260.8 & 5755.508 & -6.982 & -3.179 & 5742 \\ 261 & 5758.705 & -7.026 & -3.562 & 5746 \\ 261.2 & 5761.985 & -7.053 & -3.286 & 5751 \\ 261.4 & 5765.347 & -6.892 & -3.131 & 5756 \\ 261.6 & 5768.787 & -6.933 & -3.018 & 5760 \\ 261.8 & 5772.301 & -6.764 & -2.946 & 5765 \\ 262 & 5775.889 & -6.812 & -3.082 & 5769 \\ 262.2 & 5779.545 & -6.775 & -3.338 & 5774 \\ 262.4 & 5783.268 & -6.88 & -3.591 & 5778 \\ 262.6 & 5787.055 & -6.932 & -3.678 & 5783 \\ 262.8 & 5790.902 & -7.155 & -3.387 & 5787 \\ 263 & 5794.806 & -6.971 & -3.326 & 5792 \\ 263.2 & 5798.765 & -7.083 & -2.83 & 5796 \\ 263.8 & 5802.776 & -7.119 & -2.845 & 5801 \\ & 5806.835 & -6.587 & -2.81 & 5806 \\ & 5810.941 & -6.789 & -3.154 & 5810 \\ 5815.089 & -6.791 & -3.303 & 5815\end{array}$




\begin{tabular}{|c|c|c|c|c|}
\hline 264.2 & 5819.276 & -6.694 & -3.65 & 5819 \\
\hline 264.4 & 5823.501 & -6.859 & -3.985 & 5824 \\
\hline 264.6 & 5827.76 & -6.513 & -4.136 & 5828 \\
\hline 264.8 & 5832.05 & -6.739 & -4.091 & 5833 \\
\hline 265 & 5836.368 & -6.908 & -3.706 & 5837 \\
\hline 265.2 & 5840.711 & -7.138 & -3.823 & 5842 \\
\hline 265.4 & 5845.076 & -7.291 & -4.096 & 5846 \\
\hline 265.6 & 5849.46 & -7.257 & -4.385 & 5851 \\
\hline 265.8 & 5853.861 & -7.202 & -4.83 & 5856 \\
\hline 266 & 5858.275 & -7.314 & -4.867 & 5860 \\
\hline 266.2 & 5862.699 & -7.196 & -4.121 & 5865 \\
\hline 266.4 & 5867.131 & -7.174 & -3.733 & 5869 \\
\hline 266.6 & 5871.567 & -7.199 & -3.677 & 5874 \\
\hline 266.8 & 5876.005 & -7.318 & -3.713 & 5878 \\
\hline 267 & 5880.442 & -7.327 & -3.809 & 5883 \\
\hline 267.2 & 5884.874 & -7.228 & -3.679 & 5887 \\
\hline 267.4 & 5889.298 & -7.159 & -3.542 & 5892 \\
\hline 267.6 & 5893.713 & -7.295 & -3.506 & 5896 \\
\hline 267.8 & 5898.114 & -7.007 & -3.333 & 5901 \\
\hline 268 & 5902.5 & -7.197 & -3.825 & 5906 \\
\hline 268.2 & 5906.866 & -7.244 & -4.112 & 5910 \\
\hline 268.4 & 5911.21 & -7.278 & -3.758 & 5915 \\
\hline 268.6 & 5915.529 & -7.462 & -3.81 & 5919 \\
\hline 268.8 & 5919.82 & -7.559 & -3.799 & 5924 \\
\hline 269 & 5924.081 & -7.486 & -4.204 & 5928 \\
\hline 269.2 & 5928.307 & -7.446 & -4.072 & 5933 \\
\hline 269.4 & 5932.497 & -7.046 & -3.756 & 5937 \\
\hline 269.6 & 5936.647 & -6.722 & -3.558 & 5942 \\
\hline 269.8 & 5940.754 & -6.548 & -2.996 & 5946 \\
\hline 270 & 5944.816 & -6.934 & -3.057 & 5951 \\
\hline 270.2 & 5948.829 & -7.503 & -3.36 & 5956 \\
\hline 270.4 & 5952.791 & -7.523 & -3.5 & 5960 \\
\hline 270.6 & 5956.698 & -7.602 & -3.705 & 5965 \\
\hline 270.8 & 5960.547 & -7.835 & -3.802 & 5969 \\
\hline 271 & 5964.337 & -7.759 & -3.99 & 5974 \\
\hline 271.2 & 5968.063 & -7.968 & -4.353 & 5978 \\
\hline 271.4 & 5971.723 & -7.549 & -4.208 & 5983 \\
\hline 271.6 & 5975.314 & -7.316 & -4.385 & 5987 \\
\hline 271.8 & 5978.832 & -7.25 & -4.62 & 5992 \\
\hline 272 & 5982.275 & -7.533 & -4.603 & 5996 \\
\hline 272.2 & 5985.641 & -7.797 & -4.293 & 6001 \\
\hline 272.4 & 5988.925 & -7.769 & -3.872 & 6005 \\
\hline 272.6 & 5992.135 & -7.864 & -3.747 & 6009 \\
\hline 272.8 & 5995.327 & -7.465 & -3.474 & 6011 \\
\hline 273 & 5998.51 & -7.594 & -3.565 & 6013 \\
\hline 273.2 & 6001.684 & -7.588 & -3.598 & 6016 \\
\hline 273.4 & 6004.852 & -7.757 & -4.134 & 6018 \\
\hline 273.6 & 6008.012 & -7.807 & -4.277 & 6020 \\
\hline 273.8 & 6011.167 & -7.443 & -4.161 & 6023 \\
\hline 274 & 6014.316 & -7.239 & -4.311 & 6025 \\
\hline
\end{tabular}


PATA-1

\begin{tabular}{|c|c|c|c|c|}
\hline DFT(mm) & $\begin{array}{c}\text { Copra_Age } \\
(\text { yr BP })\end{array}$ & $\delta^{13} \mathrm{C}(\%)$ & $\delta^{18} \mathrm{O}(\%)$ & $\begin{array}{c}\text { Iscam_Age } \\
\text { (yr BP) }\end{array}$ \\
\hline 15 & 3363.4449 & 3.15777 & -0.55671585 & \\
\hline 15.1 & 3382.5874 & 2.76499 & -0.58459325 & \\
\hline 15.2 & 3401.6701 & 2.38195 & -0.73685223 & \\
\hline 15.3 & 3420.6913 & 2.22687 & -0.88573008 & \\
\hline 15.4 & 3439.6493 & 1.99382 & -1.13396818 & \\
\hline 15.5 & 3458.5425 & 1.96476 & -1.17547973 & \\
\hline 15.6 & 3477.3692 & 1.59918 & -1.22699739 & \\
\hline 15.7 & 3496.1277 & 1.43188 & -1.47885025 & 3428 \\
\hline 15.8 & 3514.8165 & 1.17652 & -1.5196057 & 3450 \\
\hline 15.9 & 3533.4338 & 0.95734 & -1.75445633 & 3473 \\
\hline 16 & 3551.978 & 1.02466 & -1.97613696 & 3495 \\
\hline 16.1 & 3570.4474 & 0.94067 & -1.92721016 & 3517 \\
\hline 16.2 & 3588.8403 & 0.64811 & -2.07128756 & 3538 \\
\hline 16.3 & 3607.1551 & 0.65025 & -1.93047122 & 3560 \\
\hline 16.4 & 3625.3902 & 0.90564 & -1.76779583 & 3581 \\
\hline 16.5 & 3643.5438 & 0.98213 & -1.81884572 & 3602 \\
\hline 16.6 & 3661.6143 & 0.65027 & -1.85463489 & 3623 \\
\hline 16.7 & 3679.6001 & 0.4409 & -1.97946858 & 3644 \\
\hline 16.8 & 3697.4994 & 0.32573 & -1.89940695 & 3664 \\
\hline 16.9 & 3715.3107 & 0.35842 & -1.98987614 & 3684 \\
\hline 17 & 3733.0322 & 0.52558 & -2.1316146 & 3704 \\
\hline 17.1 & 3750.6623 & 0.76269 & -1.990947 & 3724 \\
\hline 17.2 & 3768.1993 & 0.47614 & -1.8064824 & 3744 \\
\hline 17.3 & 3785.6417 & 1.0361 & -1.63753676 & 3763 \\
\hline 17.4 & 3802.9876 & 1.02639 & -1.79491637 & 3782 \\
\hline 17.5 & 3820.2355 & 0.82162 & -1.77506523 & 3801 \\
\hline 17.6 & 3837.3837 & 1.03875 & -2.1081257 & 3820 \\
\hline 17.7 & 3854.4305 & 0.99858 & -1.86614161 & 3838 \\
\hline 17.8 & 3871.3743 & 0.88846 & -1.80580129 & 3857 \\
\hline 17.9 & 3888.2133 & 0.75003 & -1.74073523 & 3875 \\
\hline 18 & 3904.946 & 0.76586 & -1.65407847 & 3892 \\
\hline 18.1 & 3921.5707 & 0.84449 & -1.48189193 & 3910 \\
\hline 18.2 & 3938.0858 & 0.64302 & -1.8660618 & 3927 \\
\hline 18.3 & 3954.4895 & 0.67603 & -1.70098264 & 3944 \\
\hline 18.4 & 3970.7801 & 0.43026 & -1.76301449 & 3961 \\
\hline 18.5 & 3986.9561 & 0.1503 & -2.04774919 & 3978 \\
\hline 18.6 & 4003.0158 & 0.11216 & -2.38065489 & 3994 \\
\hline 18.7 & 4018.9575 & 0.17534 & -1.76814504 & 4010 \\
\hline 18.8 & 4034.7796 & 0.04747 & -1.67693171 & 4026 \\
\hline 18.9 & 4050.4803 & 0.05985 & -1.81264336 & 4042 \\
\hline 19 & 4066.0581 & -0.00207 & -2.25758823 & 4057 \\
\hline 19.1 & 4081.5112 & 0.14103 & -2.30707962 & 4072 \\
\hline 19.2 & 4096.8381 & -0.14923 & -2.35648368 & 4087 \\
\hline 19.3 & 4112.0369 & -0.34142 & -2.59632155 & 4102 \\
\hline 19.4 & 4127.1062 & -0.2454 & -2.46896908 & 4116 \\
\hline 19.5 & 4142.0442 & -0.1991 & -2.22853134 & 4130 \\
\hline 19.6 & 4156.8492 & -0.38033 & -2.10781184 & 4144 \\
\hline
\end{tabular}




\begin{tabular}{|c|c|c|c|c|}
\hline 19.7 & 4171.5197 & -0.68902 & -2.31905841 & 4158 \\
\hline 19.8 & 4186.0538 & -0.54537 & -2.62782437 & 4171 \\
\hline 19.9 & 4200.4501 & -0.53576 & -2.67032564 & 4185 \\
\hline 20 & 4214.7068 & 0.11522 & -2.28979864 & 4197 \\
\hline 20.1 & 4228.8222 & 0.79485 & -1.84572343 & 4210 \\
\hline 20.2 & 4242.7947 & 0.62571 & -1.88333471 & 4222 \\
\hline 20.3 & 4256.6226 & 0.05748 & -2.24107254 & 4234 \\
\hline 20.4 & 4270.3043 & -0.43158 & -2.23835478 & 4245 \\
\hline 20.5 & 4283.8381 & -0.40477 & -2.39058943 & 4256 \\
\hline 20.6 & 4297.2224 & -0.10363 & -2.21119697 & 4267 \\
\hline 20.7 & 4310.4554 & 0.07912 & -2.16564483 & 4277 \\
\hline 20.8 & 4323.5356 & -0.12984 & -1.67355823 & 4287 \\
\hline 20.9 & 4336.4612 & -0.43246 & -1.88570811 & 4297 \\
\hline 21 & 4349.2306 & -0.55777 & -1.86832415 & 4307 \\
\hline 21.1 & 4361.8422 & -0.76552 & -2.24498312 & 4317 \\
\hline 21.2 & 4374.2942 & -0.4627 & -2.22130893 & 4326 \\
\hline 21.3 & 4386.585 & -0.33392 & -1.8208598 & 4336 \\
\hline 21.4 & 4398.713 & -0.58661 & -1.94080815 & 4346 \\
\hline 21.5 & 4410.6765 & -0.76248 & -2.16383287 & 4355 \\
\hline 21.6 & 4422.4738 & -0.90713 & -2.32357186 & 4365 \\
\hline 21.7 & 4434.1033 & -0.9418 & -2.5504009 & 4375 \\
\hline 21.8 & 4445.5633 & -0.85134 & -2.47327887 & 4386 \\
\hline 21.9 & 4456.8521 & -0.59112 & -2.38612503 & 4396 \\
\hline 22 & 4467.9681 & -0.36054 & -2.62296418 & 4407 \\
\hline 22.1 & 4478.9096 & -0.47817 & -2.27036699 & 4418 \\
\hline 22.2 & 4489.675 & -0.92736 & -2.70109522 & 4429 \\
\hline 22.3 & 4500.2195 & -0.87005 & -2.58673319 & 4441 \\
\hline 22.4 & 4510.5138 & -0.819 & -2.54868185 & 4453 \\
\hline 22.5 & 4520.5798 & -1.11562 & -3.03908182 & 4465 \\
\hline 22.6 & 4530.4391 & -0.91019 & -2.99640424 & 4477 \\
\hline 22.7 & 4540.1133 & -0.42448 & -2.29055231 & 4489 \\
\hline 22.8 & 4549.6244 & -0.46266 & -1.97884956 & 4501 \\
\hline 22.9 & 4558.9939 & -0.74833 & -2.42869337 & 4513 \\
\hline 23 & 4568.2435 & -0.98989 & -2.76537105 & 4525 \\
\hline 23.1 & 4577.3951 & -0.9918 & -2.97280706 & 4538 \\
\hline 23.2 & 4586.4702 & -0.78352 & -3.0546649 & 4550 \\
\hline 23.3 & 4595.4907 & -0.77896 & -3.33011007 & 4563 \\
\hline 23.4 & 4604.4782 & -0.68554 & -2.72274032 & 4576 \\
\hline 23.5 & 4613.4545 & -0.55268 & -2.4953616 & 4588 \\
\hline 23.6 & 4622.4412 & -0.5945 & -2.46750371 & 4601 \\
\hline 23.7 & 4631.46 & -0.5109 & -2.41609132 & 4614 \\
\hline 23.8 & 4640.5328 & -0.46657 & -2.33538318 & 4628 \\
\hline 23.9 & 4649.6812 & -0.34805 & -2.10649913 & 4641 \\
\hline 24 & 4658.9269 & -0.31248 & -1.74718038 & 4654 \\
\hline 24.1 & 4668.2916 & -0.29284 & -2.23347115 & 4668 \\
\hline 24.2 & 4677.7971 & 0.04659 & -2.1301879 & 4681 \\
\hline 24.3 & 4687.465 & 0.07096 & -2.11811862 & 4695 \\
\hline 24.4 & 4697.3171 & 0.16745 & -2.09452312 & 4709 \\
\hline 24.5 & 4707.3751 & 0.37622 & -1.89882022 & 4723 \\
\hline 24.6 & 4717.6607 & 0.32873 & -2.03381165 & 4737 \\
\hline 24.7 & 4728.1957 & 0.1322 & -2.07613897 & 4751 \\
\hline
\end{tabular}




\begin{tabular}{|c|c|c|c|c|}
\hline 24.8 & 4739.0016 & 0.02966 & -2.1031739 & 4766 \\
\hline 24.9 & 4750.1004 & -0.30897 & -2.44000574 & 4780 \\
\hline 25 & 4761.5135 & -0.20559 & -2.40483748 & 4795 \\
\hline 25.1 & 4773.2629 & -0.41068 & -2.3333535 & 4810 \\
\hline 25.2 & 4785.3702 & -0.62792 & -2.44818762 & 4825 \\
\hline 25.3 & 4799.0849 & -0.71175 & -2.50174871 & 4840 \\
\hline 25.4 & 4815.4154 & -0.42047 & -2.35857489 & 4857 \\
\hline 25.5 & 4834.0217 & -0.15453 & -2.21245236 & 4874 \\
\hline 25.6 & 4854.5638 & -0.30128 & -2.31832682 & 4893 \\
\hline 25.7 & 4876.7017 & -0.5161 & -2.05071823 & 4911 \\
\hline 25.8 & 4900.0955 & -0.76268 & -2.30719509 & 4930 \\
\hline 25.9 & 4924.4051 & -0.66241 & -2.55160239 & 4949 \\
\hline 26 & 4949.2906 & -1.1674 & -2.79389557 & 4969 \\
\hline 26.1 & 4974.4118 & -1.18675 & -2.90582974 & 4988 \\
\hline 26.2 & 4999.429 & -1.06719 & -2.55826868 & 5007 \\
\hline 26.3 & 5024.002 & -0.91545 & -2.44749972 & 5026 \\
\hline 26.4 & 5047.7908 & -0.7998 & -2.38333539 & 5044 \\
\hline 26.5 & 5070.4555 & -0.92024 & -2.38693565 & 5061 \\
\hline 26.6 & 5091.656 & -1.01794 & -2.17533062 & 5078 \\
\hline 26.7 & 5111.0525 & -1.30756 & -2.46347709 & 5093 \\
\hline 26.8 & 5128.3047 & -1.26197 & -2.70242399 & 5108 \\
\hline 26.9 & 5143.0729 & -0.94798 & -2.81933534 & 5121 \\
\hline 27 & 5155.017 & -0.74352 & -2.79259719 & 5132 \\
\hline 27.1 & 5165.2164 & -0.4014 & -2.4732848 & 5143 \\
\hline 27.2 & 5174.9923 & -0.23886 & -2.30989649 & 5153 \\
\hline 27.3 & 5184.367 & -0.45544 & -2.32235594 & 5163 \\
\hline 27.4 & 5193.3628 & -0.37883 & -2.37501377 & 5172 \\
\hline 27.5 & 5202.0021 & -0.04302 & -2.28981342 & 5180 \\
\hline 27.6 & 5210.3071 & -0.01669 & -2.27495933 & 5188 \\
\hline 27.7 & 5218.3003 & -0.03292 & -2.36728612 & 5196 \\
\hline 27.8 & 5226.004 & -0.02753 & -2.19175744 & 5203 \\
\hline 27.9 & 5233.4404 & -0.12572 & -2.50537026 & 5210 \\
\hline 28 & 5240.632 & 0.08095 & -2.18012055 & 5217 \\
\hline 28.1 & 5247.6011 & -0.10108 & -2.32725256 & 5224 \\
\hline 28.2 & 5254.3699 & -0.1125 & -2.29724276 & 5230 \\
\hline 28.3 & 5260.9609 & -0.20201 & -2.14739567 & 5236 \\
\hline 28.4 & 5267.3964 & -0.20897 & -2.4148113 & 5242 \\
\hline 28.5 & 5273.6987 & -0.20696 & -2.50627054 & 5248 \\
\hline 28.6 & 5279.8901 & -0.09954 & -2.42791898 & 5253 \\
\hline 28.7 & 5285.993 & -0.15542 & -2.39825591 & 5259 \\
\hline 28.8 & 5292.0298 & 0.02893 & -2.30320826 & 5264 \\
\hline 28.9 & 5298.0226 & 0.13941 & -2.24499016 & 5269 \\
\hline 29 & 5303.994 & 0.24925 & -2.25263956 & 5275 \\
\hline 29.1 & 5309.9662 & 0.20715 & -1.8929424 & 5280 \\
\hline 29.2 & 5315.9615 & 0.17191 & -1.88983459 & 5286 \\
\hline 29.3 & 5322.0023 & 0.17448 & -1.98362357 & 5291 \\
\hline 29.4 & 5328.1109 & -0.15398 & -2.11246146 & 5297 \\
\hline 29.5 & 5334.3097 & -0.67346 & -2.37345813 & 5303 \\
\hline 29.6 & 5340.621 & -0.86355 & -2.93434259 & 5308 \\
\hline 29.7 & 5347.0672 & -1.25306 & -3.18755968 & 5315 \\
\hline 29.8 & 5353.6705 & -1.49353 & -3.2830814 & 5321 \\
\hline
\end{tabular}




\begin{tabular}{ccccc}
29.9 & 5360.4533 & -1.24286 & -3.13915013 & 5328 \\
30 & 5367.4379 & -0.73343 & -2.61335409 & 5335 \\
30.1 & 5374.6467 & -0.54266 & -2.39886899 & 5342 \\
30.2 & 5382.102 & -0.79085 & -2.55046344 & 5350 \\
30.3 & 5389.8262 & -0.88963 & -2.45716303 & 5358 \\
30.4 & 5397.8415 & -0.37816 & -2.38230569 & 5366 \\
30.5 & 5406.1703 & -0.43953 & -2.80380997 & 5375 \\
30.6 & 5414.835 & -0.46214 & -2.84311929 & 5384 \\
30.7 & 5423.8578 & -0.68163 & -2.61022767 & 5394 \\
30.8 & 5433.2612 & -0.67234 & -2.45374901 & 5404 \\
30.9 & 5443.0675 & -0.95836 & -2.35905753 & 5415 \\
31 & 5453.2989 & -0.78799 & -2.17198445 & 5427 \\
31.1 & 5464.9197 & -0.75929 & -1.98163491 & 5439 \\
31.2 & 5478.7714 & -1.00116 & -2.21110253 & 5453 \\
31.3 & 5494.6918 & -1.1593 & -2.22021774 & 5468 \\
31.4 & 5512.519 & -0.71905 & -2.23872433 & 5484 \\
31.5 & 5532.0908 & -0.60295 & -2.4753568 & 5502 \\
31.6 & 5553.2453 & -0.92997 & -2.64571451 & 5520 \\
31.7 & 5575.8204 & -1.08367 & -2.16288754 & 5540 \\
31.8 & 5599.654 & -0.71507 & -2.04227474 & 5561 \\
31.9 & 5624.5842 & -0.46233 & -2.09412279 & 5583 \\
32 & 5650.4488 & -0.6604 & -2.35352064 & 5606 \\
32.1 & 5677.0859 & -0.95472 & -2.54003388 & 5630 \\
32.2 & 5704.3334 & -1.02577 & -2.89094282 & 5654 \\
32.3 & 5732.0292 & -0.8645 & -2.68100688 & 5680 \\
32.4 & 5760.0114 & -0.53415 & -2.4355268 & 5706 \\
32.5 & 5788.1178 & -0.36002 & -2.01893176 & 5734 \\
32.6 & 5816.1864 & -0.19246 & -1.91845242 & 5762 \\
32.7 & 5844.0553 & -0.0215 & -1.69309893 & 5790 \\
32.9 & 5898.5454 & -0.00108 & -2.16239233 & 5849 \\
33 & 5924.8426 & 0.3715 & -2.03293876 & 5880 \\
33.1 & 5950.2919 & 0.48624 & -1.7312555 & 5911 \\
33.2 & 5974.7311 & 0.16328 & -2.07556557 & 5943 \\
33.3 & 5997.9983 & 0.17989 & -2.00901932 & 5975 \\
33.4 & 6019.9313 & 0.41858 & -1.99931282 & 6007 \\
33.5 & 6040.3683 & 0.20066 & -2.26049528 & 6040 \\
33.6 & 6059.1471 & 0.38397 & -2.3567092 & 6073 \\
33.7 & 6076.1057 & 0.31015 & -1.86786859 & 6106 \\
33.8 & 6091.082 & 0.25471 & -1.84048782 & 6140 \\
33.9 & 6103.914 & 0.66644 & -1.62459813 & 6174 \\
34 & 6114.4397 & 1.14463 & -1.94078483 & 6208 \\
\hline & & & & \\
31.9 & & & \\
31 & & & \\
31.9 & &
\end{tabular}

